\title{
Putative Hall response of the strange metal component in $\mathrm{FeSe}_{1-x} \mathrm{~S}_{x}$
}

\author{
M. Čulo $\odot,{ }^{1,}{ }^{*}$ M. Berben, ${ }^{1}$ Y.-T. Hsu, ${ }^{1}$ J. Ayres $\odot,{ }^{2}$ R. D. H. Hinlopen $\odot,{ }^{2}$ S. Kasahara $\odot,{ }^{3, \dagger}$ Y. Matsuda, ${ }^{3}$ \\ T. Shibauchi, ${ }^{4}$ and N. E. Hussey ${ }^{1,2, \ddagger}$ \\ ${ }^{1}$ High Field Magnet Laboratory (HFML-EMFL) and Institute for Molecules and Materials, Radboud University, Toernooiveld 7 , \\ 6525 ED Nijmegen, The Netherlands \\ ${ }^{2}$ H. H. Wills Physics Laboratory, University of Bristol, Tyndall Avenue, Bristol BS8 1TL, United Kingdom \\ ${ }^{3}$ Department of Physics, Kyoto University, Sakyo-ku, Kyoto 606-8502, Japan \\ ${ }^{4}$ Department of Advanced Materials Science, University of Tokyo, Kashiwa, Chiba 277-8561, Japan
}

(Received 25 November 2020; revised 26 February 2021; accepted 22 March 2021; published 23 April 2021)

\begin{abstract}
Strange metals possess transport properties that are markedly different from those of a conventional Fermi liquid. Despite strong similarities in behavior exhibited by distinct families, a consistent description of strange metallic transport and, in particular, its evolution from low to high magnetic field strength $H$, is still lacking. The electron nematic $\mathrm{FeSe}_{1-x} \mathrm{~S}_{x}$ is one such strange metal displaying anomalous $H / T$ scaling in its transverse magnetoresistance as well as a separation of transport and Hall lifetimes at low $H$ beyond its (nematic) quantum critical point at $x_{c} \sim 0.17$. Here we report a study of the Hall response of $\mathrm{FeSe}_{1-x} \mathrm{~S}_{x}$ across $x_{c}$ in fields up to $33 \mathrm{~T}$. Upon subtraction of a normal $H$-linear component from the total Hall response (imposed by perfect charge compensation), we find a second component, ascribable to strange metal physics, that grows as $1 / T$ upon approach to the quantum critical point. Through this decomposition, we reveal that lifetime separation is indeed driven primarily by the presence of the strange metal component.
\end{abstract}

DOI: 10.1103/PhysRevResearch.3.023069

\section{INTRODUCTION}

The physical properties of many strongly correlated metals are believed to be governed, at least in part, by proximity to a quantum critical point $(\mathrm{QCP})$ where a second-order phase transition is suppressed to zero temperature by a nonthermal tuning parameter [1]. Marked deviations from standard Fermi liquid (FL) behavior are observed in the vicinity of the QCP, the most prominent being a longitudinal resistivity $\rho_{x x}(T)$ that is almost perfectly linear in temperature down to the lowest temperatures [2-4]. In some cases, this $T$ linearity also persists far beyond room temperature, in the process exceeding the Mott-Ioffe-Regel limit and contrasting sharply with the complicated $T$ dependence of the resistivity found in normal metals [5]. Such simplicity is thought to reflect some deep underlying physical principle [6] often ascribed to so-called Planckian dissipation-the maximum dissipation allowed by quantum mechanics [6,7]. Away from the QCP, $\rho_{x x}(T)$ crosses over to a quadratic $T$ dependence at low $T$, indicating the recovery of a FL ground state whose quasiparticle excitations are nonetheless dressed via their interaction with

\footnotetext{
*Matija.Culo@ru.nl

${ }^{\dagger}$ Research Institute for Interdisciplinary Science, Okayama University, 3-1-1 Tsushimanaka, Kita-ku, Okayama 700-8530, Japan

${ }^{\ddagger}$ n.e.hussey@bristol.ac.uk

Published by the American Physical Society under the terms of the Creative Commons Attribution 4.0 International license. Further distribution of this work must maintain attribution to the author(s) and the published article's title, journal citation, and DOI.
}

the critical fluctuations $[3,8]$. A characteristic fanlike phase diagram then emerges around the QCP; the upper region dominated by strange metal (SM) physics and the lower region by normal metallic (NM) behavior. In the former, quasiparticles either lose coherence or are destroyed completely, signaling a breakdown of the FL description. Many SMs also become superconducting (SC), often with a maximum transition temperature $T_{c}$ precisely where the QCP would have occurred in the absence of superconductivity. Hence, quantum criticality, SM behavior, and unconventional superconductivity are considered to be intrinsically linked.

The magnetotransport properties of SMs are also found to be distinguishable from those of their NM counterparts. The low-field Hall coefficient $R_{\mathrm{H}}$, for example, displays a strong $T$ dependence that is often interpreted as an inverse Hall angle $\cot \theta_{\mathrm{H}}$ following a distinct (invariably higher) powerlaw dependence to that of $\rho_{x x}(T)$ [9-11]. Since, according to standard Boltzmann transport theory, the $T$ dependence of both quantities is determined by a single quasiparticle lifetime, such distinct behavior in $\cot \theta_{\mathrm{H}}(T)$ and $\rho_{x x}(T)$ is often referred to as lifetime separation [9]. Although the transverse magnetoresistance (MR) is positive and quadratic at low fields, as in normal metals, its magnitude $\Delta \rho_{x x} / \rho_{x x}$ is found to scale with $\tan ^{2} \theta_{\mathrm{H}}(T)[10,12,13]$ rather than exhibit conventional Kohler scaling [i.e., with $\rho_{x x}^{-2}(T)$ ]. Both the separation of transport and Hall lifetimes and this modified Kohler's scaling of the MR have now been observed in numerous SMs, including cuprates [9,12], heavy fermions [10], and iron pnictides $[11,13]$. Given the very different Fermi surface (FS) topologies, dominant interactions, and energy scales across these various families, this striking similarity in their 
(a)

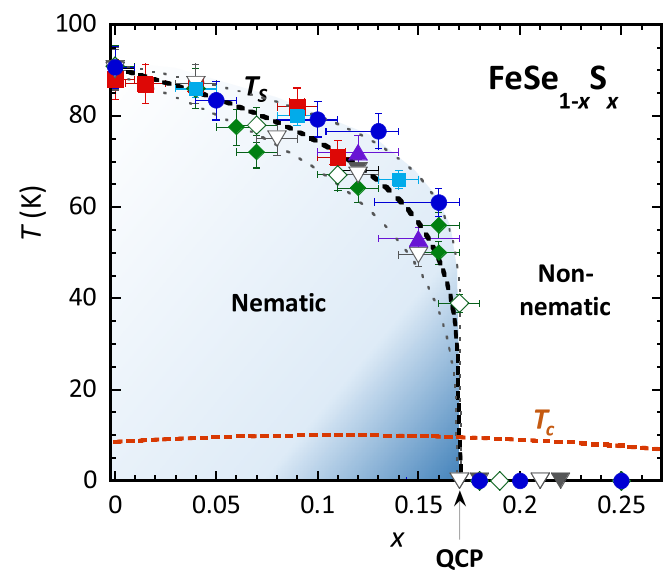

(b)

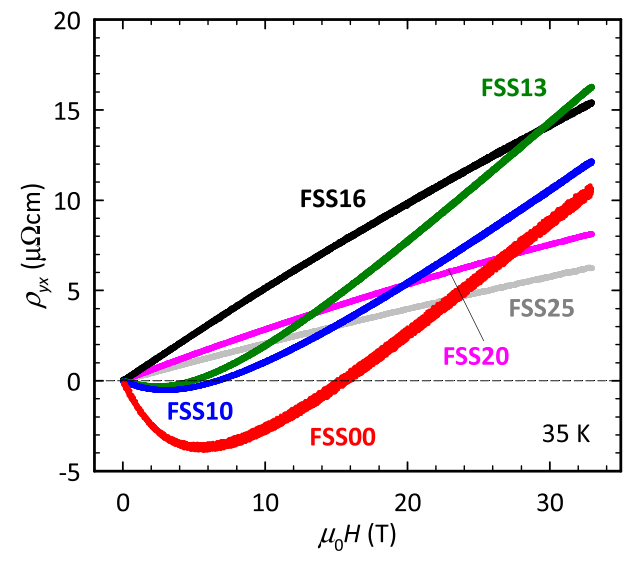

FIG. 1. (a) Phase diagram of $\mathrm{FeSe}_{1-x} \mathrm{~S}_{x}$ focusing on the evolution of $T_{s}$, the structural phase transition, as a function of $\mathrm{S}$ content [40]. Here, $T_{s}$ is estimated from the cusp in the derivative of $\rho(T)$, which in pure FeSe coincides with the midpoint of the specific heat jump [41]. The solid blue circles represent the samples used in this study. The other data points are from Refs. [42] (big red squares), [38] (closed triangles), [22] (closed inverted triangles), [28] (open inverted triangles), [23] (closed diamonds), [31] (open diamonds), and [43] (small cyan squares). The orange dashed line traces out $T_{c}(x)$. (b) Measured Hall resistivity $\rho_{y x}(H)$ up to $33 \mathrm{~T}$ at $T=35 \mathrm{~K}$ for six different nominal $x$ concentrations as labeled.

transport behavior hints once again at some universal, but as yet unidentified, organizing principle.

Recently, focus has shifted to the study of SM magnetotransport at higher field strengths, such as those provided by international facilities. Several intriguing aspects of the SM charge dynamics have emerged in the process. Arguably the most striking of these is the observation of a crossover to $H$-linear MR in systems close to a putative QCP [14-19]. The precise form of the MR respects a type of scaling in which the $H$ and $T$ dependencies appear in quadrature [14], suggesting an intimate connection to the (Planckian) $T$-linear resistivity at zero field. How this $H / T$ scaling connects, if at all, to the modified Kohler's scaling seen at lower fields is not known at present. Indeed, it might even be the case that these two types of scalings reflect the response of different conducting elements within the SM phase. In the iron chalcogenide $\mathrm{FeSe}_{1-x} \mathrm{~S}_{x}$, for example, high-field studies have revealed the presence of two additive components in the transverse MR, one following a conventional $H^{2}$ dependence, the other the quadrature scaling form [18] that evolves systematically in a manner set by proximity to the QCP. Collectively, these findings suggest a coexistence of distinct charge components in $\mathrm{FeSe}_{1-x} \mathrm{~S}_{x}$, a coexistence that has now also been inferred in both cuprates [19,20] and iron pnictides [21].

In addition to the quadrature $\mathrm{MR}, \mathrm{FeSe}_{1-x} \mathrm{~S}_{x}$ also exhibits SM characteristics at lower field, including a modified Kohler's scaling $\left(\Delta \rho_{x x} / \rho_{x x} \propto \tan ^{2} \theta_{\mathrm{H}}\right)$ and lifetime separation $\left[\cot \theta_{\mathrm{H}} \sim c_{0}+c_{2} T^{2}\right.$ throughout the entire $T$ range where $\rho_{x x}(T)$ is $T$ linear] [22]. To gain fresh insight into the origins of this behavior, we have carried out a complementary highfield study of the Hall resistivity $\rho_{y x}$ of the same crystals used in our earlier MR study [18] with a view to combining the two responses into a single unified model. By fitting the MR and Hall responses self-consistently, we find that not only $\rho_{x x}$ but also $\rho_{y x}$ comprises two components: one that is conventional and one that connects to the quadrature MR and evolves systematically across $x_{c}$. By tracking the evolution of both contributions with $T$ and $x$, a link is then established between the high-field response and the anomalous lifetime separation observed at low fields. Finally, a model is presented that seeks to reconcile the SM transport seen in $\mathrm{FeSe}_{1-x} \mathrm{~S}_{x}$ with the lack of enhancement in the effective masses of individual pockets across $x_{c}$ [23].

\section{II. $\mathrm{FeSe}_{1-x} \mathrm{~S}_{x}$}

$\mathrm{FeSe}_{1-x} \mathrm{~S}_{x}$ is unique among Fe-based superconductors in that it offers the possibility to study the distinct role of nematic QC fluctuations in both the SM and unconventional SC phases (for recent reviews, see Refs. [24-27]). Pure FeSe undergoes a nematic transition at $T_{s} \approx 90 \mathrm{~K}$ accompanied by a tetragonal-to-orthorhombic structural transition. The application of pressure or substitution of Se with $\mathrm{S}$ effectively suppresses $T_{s}$, the latter terminating at a nematic QCP at $x_{c} \approx 0.17$ [28] [see Fig. 1(a)]. While pressure enhances $T_{c}$ in FeSe, S substitution appears to have little effect on the SC transition, though the SC gap is observed to fall by at least a factor of 2 beyond the nematic phase [29,30].

Near the nematic QCP, $\rho_{x x}(T) \sim T$ down to the lowest accessible temperatures $(\sim 1.4 \mathrm{~K})$, while away from QCP, $T^{2}$ behavior is restored at low $T[22,31-33]$. On approaching the nematic QCP from the high $x$ side, the coefficient of the $T^{2}$ resistivity is found to be enhanced by at least one order of magnitude $[31,32]$. Hence, it has been suggested that nematic, rather than antiferromagnetic (AFM), critical fluctuations drive the anomalous transport in $\mathrm{FeSe}_{1-x} \mathrm{~S}_{x}$ [32], though this interpretation is still contested [34-36]. While no magnetic order is observed in $\mathrm{FeSe}_{1-x} \mathrm{~S}_{x}$ under ambient pressure, at any concentration, AFM correlations are known to develop as the temperature is lowered. Moreover, a spin density wave (SDW) phase emerges under applied pressure $p$. Crucially though, the SDW phase is found to shift to higher $p$ as $x \rightarrow$ $x_{c}$ [37], while beyond $x=0.09$, AFM correlations become weaker [38]. Hence, it seems unlikely that spin fluctuations, 
though present, are responsible for the QC-like transport seen in $\mathrm{FeSe}_{1-x} \mathrm{~S}_{x}$.

\section{EXPERIMENT}

A series of $\mathrm{FeSe}_{1-x} \mathrm{~S}_{x}$ single crystals were grown in Kyoto by the chemical vapor transport technique [32]. All samples were cut into thin rectangular plates of typical dimensions $0.1 \times 0.5 \times 1.5 \mathrm{~mm}^{3}$, the largest surface being the crystallographic $a b$ plane. One pair of current and two pairs of Hall contacts were then made by applying indium solder and silver paint directly onto the lateral sides of each plate to ensure current flow throughout the sample. Low-field Hall effect measurements were performed on a set of single crystals with nominal $x$ values of $0.00,0.05,0.10,0.13,0.16$, $0.18,0.20$, and 0.25 (labeled hereafter FSS00, FSS05, FSS10, FSS13, FSS16, FSS18, FSS20 and FSS25) in a cryogenic cryogen free measurement system with a $9 \mathrm{~T} \mathrm{SC}$ magnet over the temperature range $T_{c}<T<300 \mathrm{~K}$. At each temperature, the Hall voltage $V_{y}$ was measured by a standard ac lock-in technique and antisymmetrized to eliminate any longitudinal component. The Hall resistivity $\rho_{y x}=V_{y} t / I_{x}$ while $R_{\mathrm{H}}=\rho_{y x} /\left(\mu_{0} H\right)$, where $t$ is the sample thickness, $I_{x}=I$ the applied current, and $\mu_{0} H$ the applied field. The evolution of $R_{\mathrm{H}}(x)$ reveals a gradual but nonetheless marked change across $x_{c}$ [39]. Specifically, the strong nonlinearity in $\rho_{y x}(H)$ that is observed within the nematic phase disappears above $T_{s}$ and is gradually weakened with increasing $x$, while its $H$ dependence (more specifically, the sign of the second derivative $\left.d^{2} \rho_{y x} / d H^{2}\right)$ becomes inverted. The overall reduction in $R_{\mathrm{H}}$ beyond $x_{c}$ is consistent with our earlier study [22].

The high-field measurements were carried out on the same samples (barring the $x=0.05$ and 0.18 samples) in a resistive magnet at the High Field Magnet Laboratory (Nijmegen) with a maximum field of $33 \mathrm{~T}$. For all samples, an ac current of $0.5-1 \mathrm{~mA}$ was applied, $I \| a b$ with $H \| c$, as determined

(a)

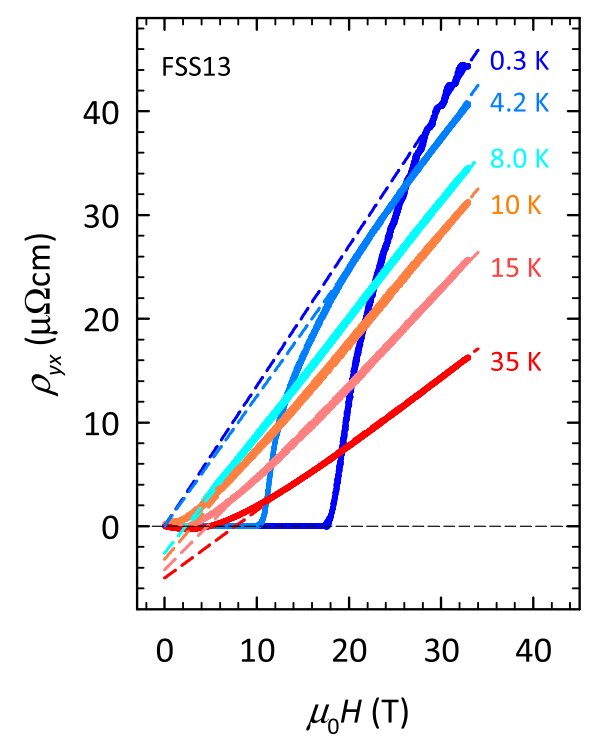

(b)

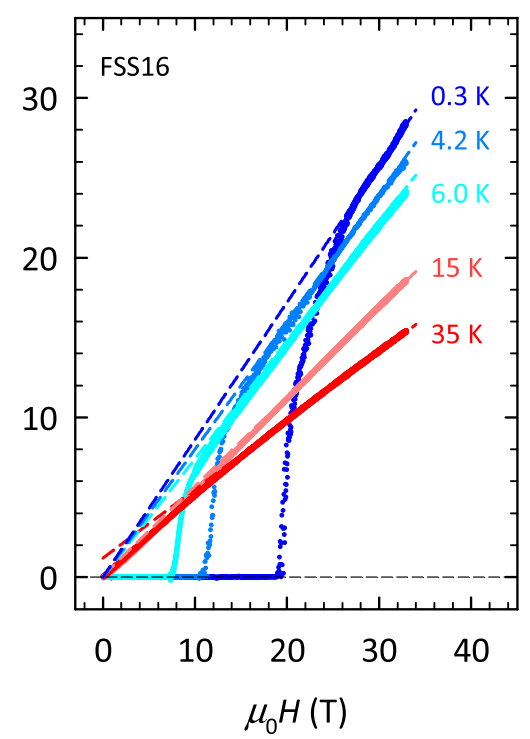

using a Hall probe mounted onto the rotating sample platform. Measurements were performed from -33 to $+33 \mathrm{~T}$ at fixed temperatures between $0.3 \mathrm{~K}$ and $35 \mathrm{~K}$. Stable temperatures above $4.2 \mathrm{~K}$ were achieved using a constant heater power between 1.2 and $4.2 \mathrm{~K}$ by putting the sample space in good thermal contact with a He-4 bath at various constant pressures, and below $1.2 \mathrm{~K}$ by using constant pressures of He-3 liquefied inside the sample space.

\section{RESULTS AND ANALYSIS}

\section{A. Hall data}

Figure 1(b) shows $\rho_{y x}(H)$ at $35 \mathrm{~K}$ for all samples measured up to $33 \mathrm{~T}$. Qualitatively similar results for pure FeSe were reported elsewhere [44]. In all cases, the Hall effect is hole dominated at high field with a change of sign in the nematic phase at low fields for $x<0.16$. Beyond $x=0.16$, the slope gradually decreases as one moves further away from the nematic QCP. The $T$ dependence of $\rho_{y x}(H)$ below $35 \mathrm{~K}$ is shown in Fig. 2 for three representative $x$ values: to the left of the QCP (FSS13, left panel), close to $x_{c}$ (FSS16, middle panel), and to the right of the QCP (FSS25, right panel). In the nematic phase, the low- $T$ response is proportional to $H$, in contrast to what is observed at more elevated temperatures, whereas for $x>x_{c}$, it is the high- $T$ response that is $H$ linear. Near the QCP itself, $\rho_{y x}(H)$ is approximately $H$ linear at all temperatures [as shown in Fig. 2(b), the high-field linear slopes for FSS16 extrapolate close to the origin at all temperatures]. Together, the form of $\rho_{y x}(H, T, x)$ suggests a complex but nonetheless systematic evolution of the Hall response across the phase diagram. The sweep at $T=0.3 \mathrm{~K}$ in FSS13 also shows the onset of quantum oscillations (QOs) with a frequency $F \sim 740 \pm 30 \mathrm{~T}$, in good agreement with that reported previously [23].

\section{(c)}

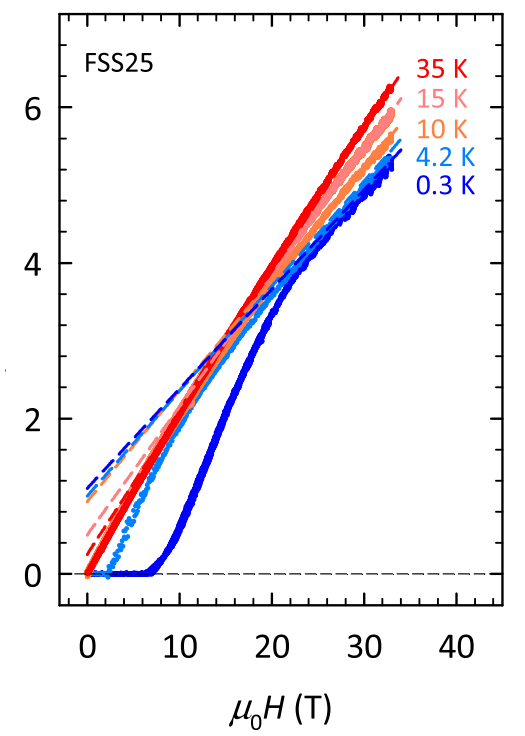

FIG. 2. Temperature evolution of $\rho_{y x}(H)$ in $\mathrm{FeSe}_{1-x} \mathrm{~S}_{x}$ for (a) FSS13, i.e., to the left of the nematic QCP, (b) FSS16, near the QCP, and (c) FSS25, beyond the QCP. Dashed lines are linear fits to the high-field data (above $25 \mathrm{~T}$ ). 


\section{B. Two-band and three-band analysis}

The layered crystal structure of FeSe ensures that bands derived from the $\mathrm{Fe} d$ orbitals have a quasi-2D character $[24,25]$. While a determination of the FS topology and, in particular, the number of electron and hole pockets, has proved controversial, a level of consistency has nonetheless begun to emerge. Angle-resolved photoemission (ARPES) studies on detwinned FeSe [45-47], as well as nano-ARPES studies on single domains [48], have concluded that only one electron and one hole pocket survives within the nematic phase. This is consistent with independent analysis $[32,41]$ of QOs $[49,50]$ and specific heat data $[41,51,52]$. The possibility of a second small electron pocket-inferred from mobility spectrum analysis of Hall and MR data [44,53]—has not been completely ruled out. It is noted, however, that no clear evidence for such a pocket has yet been seen in QO experiments, despite the fact that such a small, light, and highly mobile pocket ought to be easy to detect.

As $\mathrm{Se}$ is replaced with $\mathrm{S}$, the size of each pocket increases while preserving the compensation condition [23]. There is some evidence for an additional QO frequency possibly emerging, and then vanishing again, within a narrow range $(0.11<x<0.17)$, though the same data set has been interpreted by another group as indicating the appearance of a new frequency (pocket) only beyond $x_{c}$ [54]. Hence, the precise FS topology of $\mathrm{FeSe}_{1-x} \mathrm{~S}_{x}$ is not yet established. Nevertheless, it seems reasonable to conclude that there are at least two but no more than three distinct pockets in $\mathrm{FeSe}_{1-x} \mathrm{~S}_{x}$ for $0 \leqslant x \leqslant$ 0.25 and so, in the following, we first attempt to fit our Hall effect data using standard two- and three-band models.

Each model provides expressions for $\rho_{x x}(H)$ and $\rho_{y x}(H)$ that depend on the electron $\left(n_{e}, \mu_{e}\right)$ and hole $\left(n_{h}, \mu_{h}\right)$ densities and mobilities. These expressions are intimately related through one or more common fitting parameters [55] and, to perform multiband modeling effectively, it is imperative to fit both $\rho_{y x}(H)$ and $\rho_{x x}(H)$ simultaneously. A three-band model using standard matrix formalism [56] was applied previously to explain the low-field $\rho_{x x}(H)$ and $\rho_{y x}(H)$ data in pure FeSe up to $14 \mathrm{~T}$ [44] under the assumption that $n_{e}=n_{h}$. In our analysis, we have relaxed this condition to maximize the flexibility of our fitting. Even so, we found it impossible to simultaneously fit $\rho_{x x}(H)$ and $\rho_{y x}(H)$ over the full field range up to $33 \mathrm{~T}$ using either the two-band model (for all samples) or the three-band model (for $x<0.16$ ) [57].

One particular challenge was that for $x<0.16, \rho_{y x}(H)$ changes sign at low fields, while over the same field range, $\rho_{x x}(H)$ exhibits simple and rather universal behavior, namely, $H^{2}$ at low fields and $H+H^{2}$ at high fields [18]. A similar MR response was observed in pure and doped $\mathrm{BaFe}_{2} \mathrm{As}_{2}$ [58-60] where a nonsaturating linear MR and highly nonlinear $\rho_{y x}(H)$ were attributed to the presence of Dirac cone states [61]. There have also been several reports claiming evidence for Dirac cones in $\mathrm{FeSe}_{1-x} \mathrm{~S}_{x}$, though only inside the nematic phase [53,62,63]. Crucially, however, the $H$-linear component in the MR in $\mathrm{FeSe}_{1-x} \mathrm{~S}_{x}$ is found to extend beyond the nematic phase [18], implying that such Dirac-like states, as part of an extended three-band model, cannot be the source of the anomalous magnetotransport in $\mathrm{FeSe}_{1-x} \mathrm{~S}_{x}$. Of course, one could always add further complexity to the model, e.g., by considering any curvature in the $\mathrm{FS}(\mathrm{s})$ or the presence of anisotropic scattering or effective masses, all of which have been detected in FeSe [64]. To the best of our knowledge, however, no variant of these multiband models could account for the observation [18] of a purely quadrature MR-with precise $H / T$ scaling - in a sample beyond $x_{c}$ in which the usual orbital MR had been effectively quenched by disorder. This observation alone compels us to explore an alternative scenario, one in which the SM plays a central role.

\section{Normal and strange metal conductivity in $\mathrm{FeSe}_{1-x} \mathbf{S}_{x}$}

The previous high-field MR study [18] showed convincingly that the transverse MR could be decomposed into two distinct contributions, one that exhibited quadrature scaling characteristic of other SMs, and one that followed a strict $H^{2}$ dependence without saturation up to $33 \mathrm{~T}$ [65]. Such a delineation provides strong evidence for the presence of two separate contributions to the conductivity. How these two components combine, i.e., through adding conductivities or resistivities, is not known precisely, but in our analysis, we find it more revealing to express the total longitudinal $\left(\sigma_{x x}^{\text {tot }}\right)$ and Hall $\left(\sigma_{y x}^{\text {tot }}\right)$ conductivities as a sum of NM and SM components. In this case, the MR becomes a weighted sum of the two independent contributions. To separate the contributions of the NM and SM component to the total Hall conductivity $\sigma_{y x}^{\text {tot }}$, we have adopted the following strategy: (i) calculate $\sigma_{y x}^{\text {tot }}(H)$ from the experimentally determined quantities $\rho_{x x}^{\text {tot }}(H)$ and $\rho_{y x}^{\text {tot }}(H)$, (ii) fit the calculated $\sigma_{y x}^{\text {tot }}(H)$ assuming the presence of only the NM component (in the high-field limit), and (iii) ascribe the residuals $\sigma_{y x}^{\text {tot }}-\sigma_{y x}^{\mathrm{NM}}$ of such a fitting procedure to the SM component.

Figure 3 shows the total Hall conductivities (black dots) for the same three $15 \mathrm{~K}$ sweeps depicted in Fig. 2 (this temperature is chosen as it lies outside the SC fluctuation regime). In all cases, $\sigma_{y x}^{\text {tot }}(H)$ has a minimum at intermediate fields, while FSS13 also has a small maximum at low $H$.

Before proceeding, it is important to recognize that the nonsaturating $H^{2} \mathrm{MR}$ of the NM response [18] can only arise if the charge is fully compensated. This in turn imposes a strong constraint on the form of $\rho_{y x}^{\mathrm{NM}}(H)$-namely, a strict $H$-linear dependence-and allows us to consider the bipolar contributions to $\rho_{y x}^{\mathrm{NM}}(H)$ as a single entity (weighted by the difference in their mobilities). Then, within a parallel channel scenario, $\sigma_{y x}^{\text {tot }}(H)$ becomes a sum of the NM and SM components, the former assuming a specific form imposed by the compensation condition [67]:

$$
\sigma_{y x}^{\mathrm{NM}}(H)=\frac{-a \mu_{0} H}{\left[b \rho_{x x}^{\text {tot }}(0)+b^{2} \beta_{\mathrm{NM}}\left(\mu_{0} H\right)^{2}\right]^{2}+\left[a \mu_{0} H\right]^{2}} .
$$

Here, $a$ is a free fitting parameter related to the linear slope of $\rho_{y x}^{\mathrm{NM}}(H)\left(=R_{\mathrm{H}}\right), \rho_{x x}^{\text {tot }}(0)$ is the measured zero-field resistivity [18], $\beta_{\mathrm{NM}}$ is the (as-measured) quadratic MR term [18], and $b=\rho_{x x}^{\mathrm{NM}}(0) / \rho_{x x}^{\text {tot }}(0)$ is a second free fitting parameter [68].

The second contribution to $\sigma_{y x}^{\text {tot }}(H)$ is assumed, rightly or wrongly, to originate from the same component that generates the quadrature MR. The scale invariance of this quadrature MR ties it directly to the (zero-field) $T$-linear resistivity that is itself associated with Planckian dissipation. In cuprates, the quadrature MR exhibits nonorbital character [19] and, as 
(a)

(b)

(c)
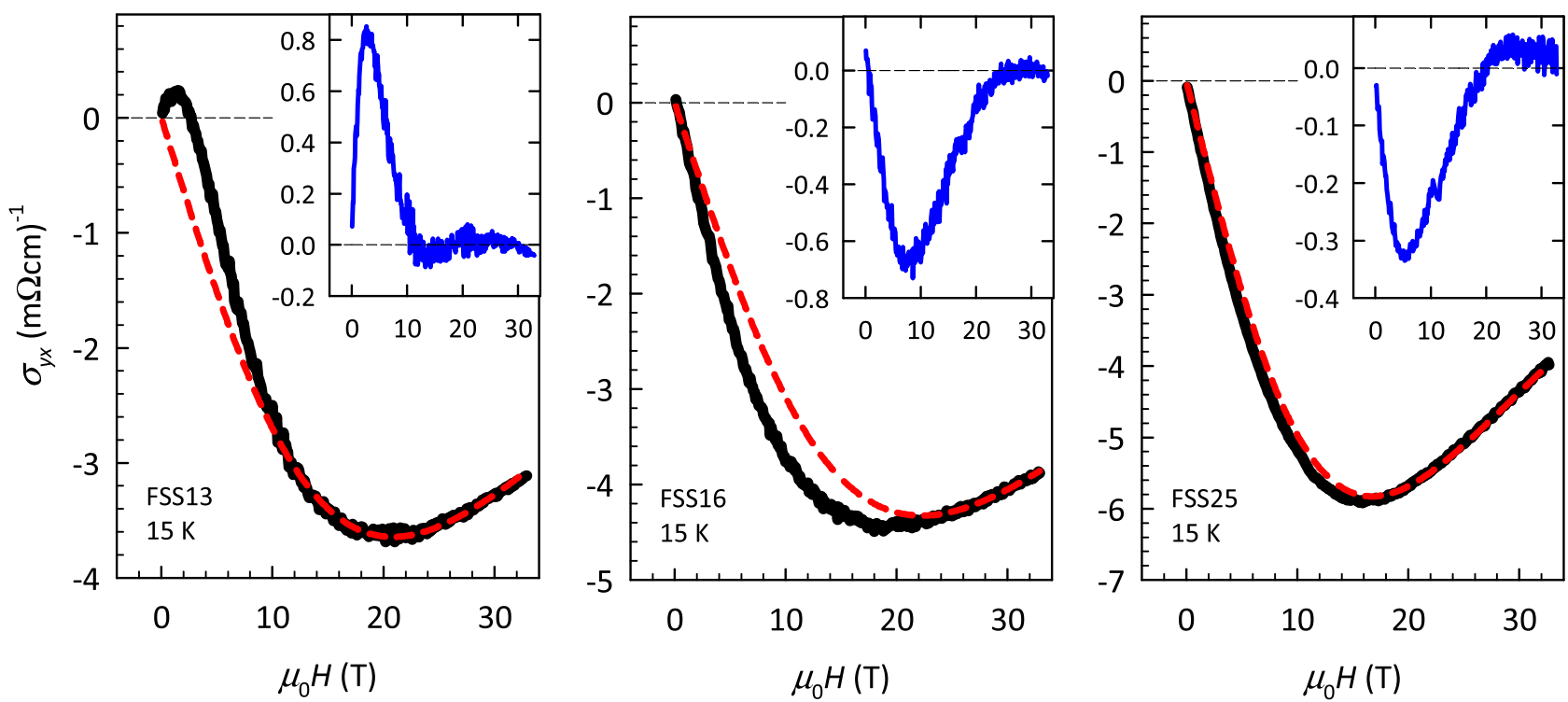

FIG. 3. Fitting of the calculated Hall conductivity $\sigma_{y x}(H)$ in $\operatorname{FeSe}_{1-x} S_{x}$ using Eq. (1). $\sigma_{y x}(H)$ at $15 \mathrm{~K}$ for the same three concentrations shown in Fig. 2 (a) FSS13, (b) FSS16 and (c) FSS25. Black lines represent the measured data [66] and red dashed lines represent best fits to Eq. (1) in the high-field regime (see text), assuming only a NM component. (The field range used in the fitting procedure is 15-33 T, 25-33 T, and 20-33 T for FSS13, FSS16, and FSS25, respectively.) The residuals representing the Hall conductivity of the SM component are shown in the insets.

such, is not expected to generate a simultaneous Hall conductivity [20]. In pnictides, on the other hand, the quadrature MR has been attributed to anisotropic (hot spot) scattering of quasiparticles [69] that will eventually become suppressed at the highest fields. Thus, in either scenario, one expects $\sigma_{y x}^{\mathrm{SM}}(H) \rightarrow 0$ as $H$ increases and, in a similar spirit, we proceed by attempting to fit $\sigma_{y x}^{\text {tot }}(H)$ to Eq.(1) by biasing the fit to the high-field region of the experimental curve where we expect $\sigma_{y x}^{\mathrm{SM}}(H)$ to vanish [70].

The resultant fits are shown as red dashed lines in Fig. 3. While this fitting routine is deliberately constrained, it has a number of advantages: (i) it relies only on as-measured quantities $\left[\rho_{x x}(H)\right.$ and $\left.\rho_{y x}(H)\right]$, (ii) it has only two free parameters, and (iii) it is naturally self-consistent [71]. The residuals of each fit, ascribed then to $\sigma_{y x}^{\mathrm{SM}}(H)$, are shown in the insets. Two features of these residuals are of note; the change of sign in $\sigma_{y x}^{\mathrm{SM}}(H)$ that occurs around $x=0.13$ and the goodness of fit for FSS25. Intriguingly, the sign change mirrors that seen in the low- $T$ nematic susceptibility at around $x=0.12$ [28], possibly indicating a reversal in the anisotropy of the dominant scattering process. The goodness of fit for FSS25 is consistent with the expectation that the electronic ground state in $\mathrm{FeSe}_{1-x} \mathrm{~S}_{x}$ will eventually become isotropic with increasing $x$. Nevertheless, even for FSS25 the SM component survives, as evidenced by the nonlinearity in $\rho_{y x}(H)$ (see Fig. 1) as well as the persistence of a small, yet finite quadrature term in the MR [18].

\section{Strange metal component}

The corresponding Hall resistivity $\rho_{y x}^{\mathrm{SM}}(H)$ is obtained from the residual $\sigma_{y x}^{\mathrm{SM}}(H)$ curves [72]. Figure 4 shows $\rho_{y x}^{\mathrm{SM}}(H)$ at representative temperatures for all $x>0$. As indi- cated by the solid lines in Fig. $4, \rho_{y x}^{\mathrm{SM}}(H)$ can be fitted well to the empirical expression $\rho_{y x}^{\mathrm{SM}}(H)=c \mu_{0} H \exp \left[-d\left(\mu_{0} H\right)^{2}\right]$, where $c$ and $d$ are free parameters [73]. Despite the various assumptions made in extracting $\rho_{y x}^{\mathrm{SM}}(H)$, its overall evolution with $x$ appears to correlate well with that of the zero-field resistivity across the nematic QCP [32]. For FSS10 and FSS13, for example, $\rho_{y x}^{\mathrm{SM}}(H)$ progressively decreases with decreasing $T$. This is especially evident from the $10 \mathrm{~K}$ curve for FSS13 [Fig. 4(b)]. A similar $T$ dependence is also observed in FSS25 [Fig. 4(e)]. Thus, on either side of the QCP, the low- $T$ Hall response appears to be dominated by the NM component. For FSS16 and FSS20, on the other hand, $\rho_{y x}^{\mathrm{SM}}(H)$ grows as $T$ decreases, indicating a strengthening of the SM component as the temperature is reduced.

\section{E. Normal metal component}

Given that $\mathrm{FeSe}_{1-x} \mathrm{~S}_{x}$ is presumed here to be a compensated two-band semimetal, the parameters $n, \mu_{e}$ and $\mu_{h}$ can be obtained directly from a self-consistent analysis of the three extracted quantities for the normal metal component: $\rho_{x x}^{\mathrm{NM}}(T)$, $R_{\mathrm{H}}^{\mathrm{NM}}$ and $\Delta \rho_{x x}^{\mathrm{NM}} / \rho_{x x}^{\mathrm{NM}}$ [74]. The mobilities exhibit a metallic $T$ dependence and fall in the range $200-1000 \mathrm{~cm}^{2} / \mathrm{Vs}$ with $\mu_{h}>\mu_{e}$ for all $T$ and $x$ studied. The carrier densities range from 2 to $4 \times 10^{20} \mathrm{~cm}^{-3}(0.015-0.03$ per Fe atom) and are constant, to within our experimental uncertainty, across the studied temperature range. The variation of $n(x)$ per Fe across the series is compared in Fig. 5 with estimates extracted from QO experiments [23]. Curiously, the densities determined from our two-carrier analysis of the NM component are found to be approximately half those derived from the QO study. We shall return to this point in the following section. 


\section{(a)}

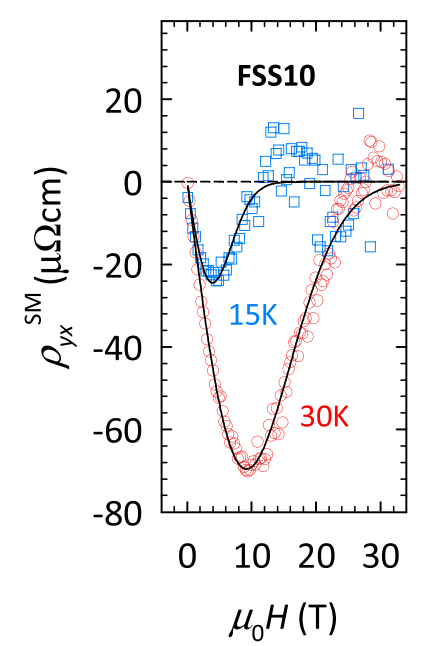

(b)

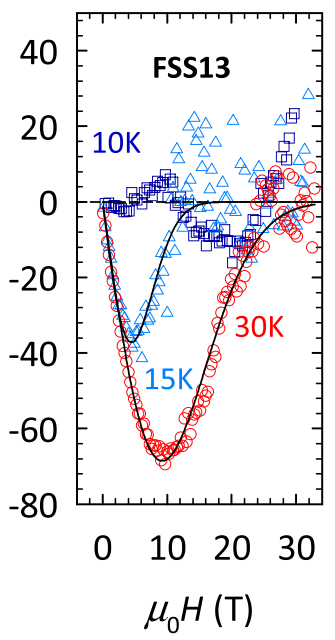

(c) (d)

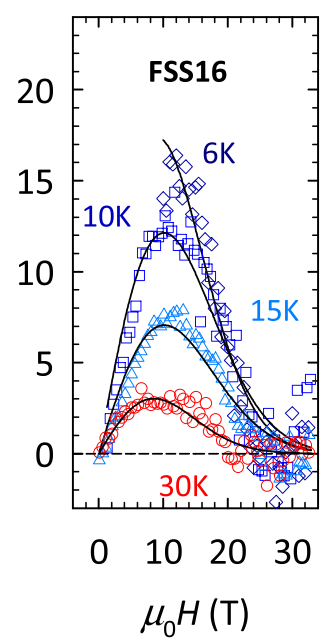

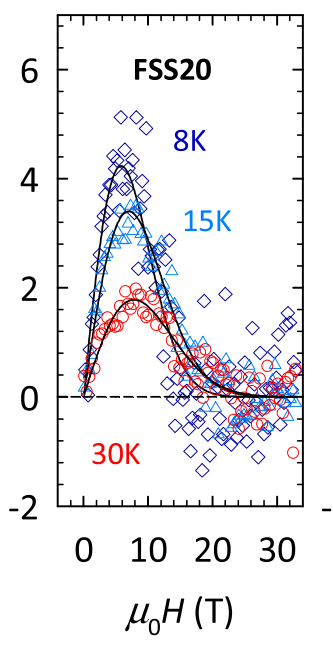

(e)

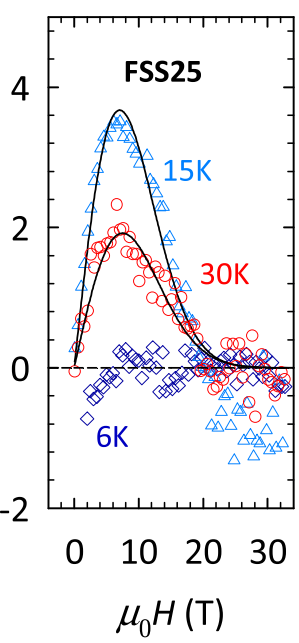

FIG. 4. Hall response of the SM component in $\mathrm{FeSe}_{1-x} \mathrm{~S}_{x}$. Shown are the Hall resistivities of the SM component $\rho_{y x}^{\mathrm{SM}}(H)$ for (a) FSS10, (b) FSS13, (c) FSS16, (d) FSS20, and (e) FSS25 at temperatures where the low-field feature was not completely screened by superconductivity. Open symbols represent the data while full lines represent the fits to the empirical relation $\rho_{y x}^{\mathrm{SM}}(H)=c \mu_{0} H \exp \left[-d\left(\mu_{0} H^{2}\right)\right][72]$. The increased scatter in $\rho_{y x}^{\mathrm{SM}}(H)$ at high fields, not visible in $\sigma_{y x}^{\mathrm{SM}}$ (see insets of Fig. 3), results from the transformation of $\sigma_{y x}^{\mathrm{SM}}$ to $\rho_{y x}^{\mathrm{SM}}$ using Eq. (F1) and the diminishing magnitude of $\sigma_{x x}^{\mathrm{SM}}$ with increasing field. Scatter in the data at 10 and $15 \mathrm{~K}$ for FSS10 and FSS13 may be caused by superconducting fluctuations in vicinity of $T_{c}$.

\section{DISCUSSION}

\section{A. Comparison with magnetoresistance}

The analysis presented above supports the notion, put forward in Ref. [18], that the dc conductivity of $\mathrm{FeSe}_{1-x} \mathrm{~S}_{x}$

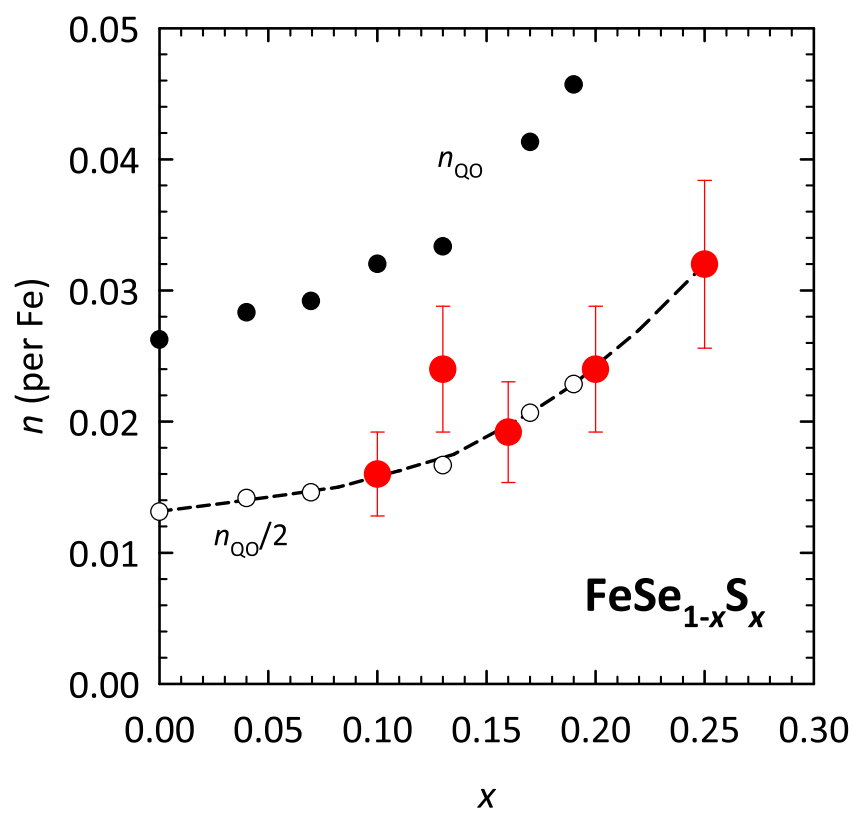

FIG. 5. Carrier density of the NM component across the series. Large closed circles show the average value for $n$ extracted using the compensated two-band model at different temperatures [74]. Small closed circles are extracted from QO frequencies [23] (averaged frequencies for both pockets maintaining compensation condition; see Ref. [32] for details). Open circles are the same $n$ values extracted from QO frequencies halved. Black dashed line is a guide to the eye.
$(0 \leqslant x \leqslant 0.25)$ contains contributions from both a NM component (which itself is compensated) and a SM component of, as yet, unknown origin. Moreover, as shown in Fig. 6, the evolution of the Hall $\left[\rho_{y x}^{\mathrm{SM}}(T, H, x)\right.$-red circles] and longitudinal $\left[\Delta \rho_{x x}^{\mathrm{SM}}(T, H, x)\right.$-blue squares] resistivities across the series exhibit clear parallels. For FSS16, both quantities increase significantly, by a factor of 3-6 below $30 \mathrm{~K}$, while for FSS20, the enhancement is reduced. Further away from the QCP, these ratios typically fall upon leaving the QC fan (gray shading in Fig. 6), vanishing inside the regime (shaded white) where $T^{2}$ resistivity is restored. This implies that all anomalous signatures of SM transport do indeed disappear inside the FL regime.

A recent high-field study carried out on $\mathrm{BaFe}_{2}\left(\mathrm{As}_{1-x} \mathrm{P}_{x}\right)_{2}$ also revealed a Hall response comprising both a NM and a SM component, the latter peaking close to the (AFM) QCP [21]. In their analysis, the authors added Hall coefficients rather than Hall conductivities. Although, in principle, a similar serial conductivity analysis could be applied to $\mathrm{FeSe}_{1-x} \mathrm{~S}_{x}$, such a procedure would be inconsistent with the analysis of the corresponding MR data reported in Ref. [18] whose decomposition into SM and NM components was carried out assuming parallel conductivity channels. Furthermore, the serial picture would require a nonlinear Hall resistivity of the NM component that would conflict with the $H^{2}$ contribution of the NM component to the total MR and the corresponding requirement for charge compensation [18]. Therefore, we find adding Hall conductivities to be the more appropriate model for $\mathrm{FeSe}_{1-x} \mathrm{~S}_{x}$. Nevertheless, the fact that $\rho_{y x}^{\mathrm{SM}}(H)$ in $\mathrm{BaFe}_{2}\left(\mathrm{As}_{1-x} \mathrm{P}_{x}\right)_{2}$ is found to have a similar field profile to the one in $\mathrm{FeSe}_{1-x} \mathrm{~S}_{x}$, with a maximum at relatively low $H$ followed by an exponential decay at higher fields [21], suggests once again that the Hall response of different SMs is qualitatively the same. 


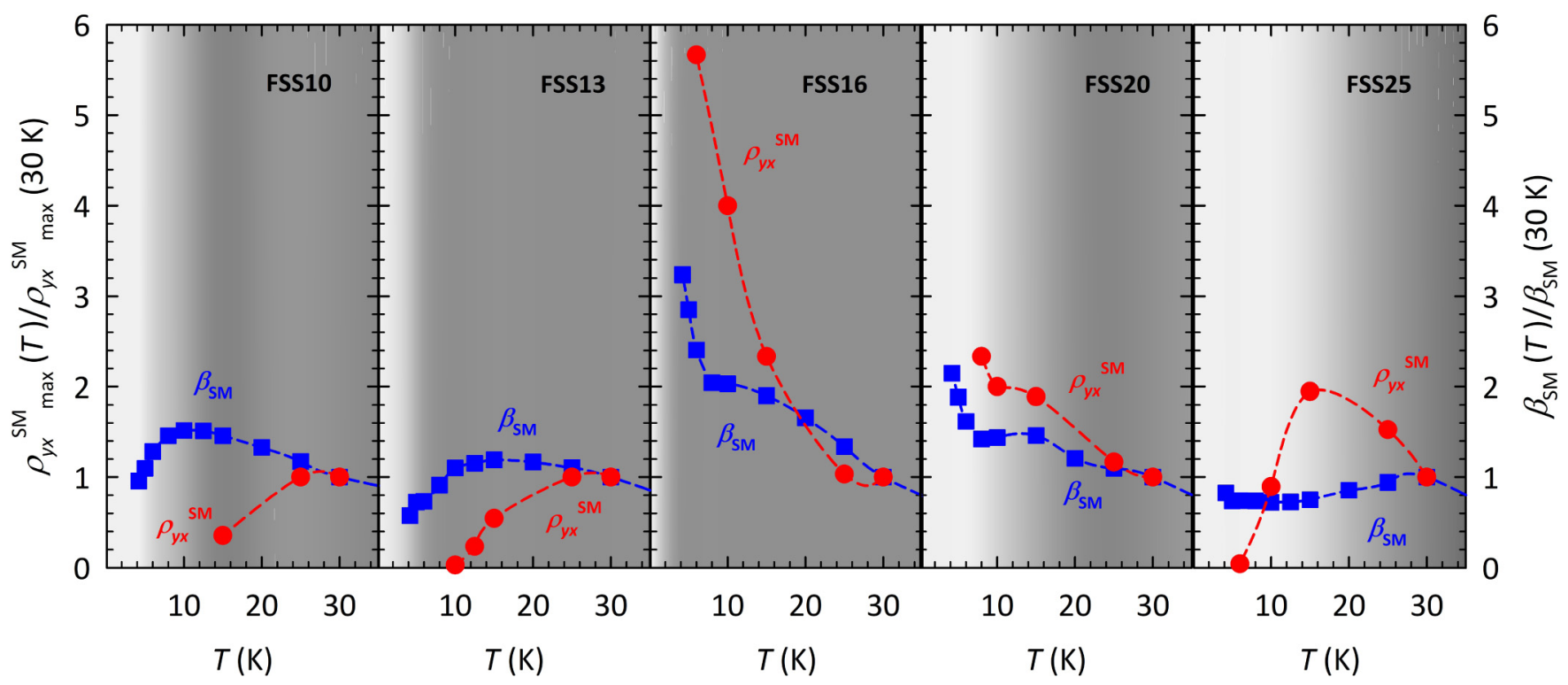

FIG. 6. Magnetotransport properties of the $\mathrm{SM}$ component in $\mathrm{FeSe}_{1-x} \mathrm{~S}_{x}$. Red circles show the $T$ dependence of the maximum in $\rho_{y x}^{\mathrm{SM}}(H)$ normalized to its value at $30 \mathrm{~K}$ (left axis) and blue squares represent $\beta_{\mathrm{SM}}$, the slope of the $H$-linear MR from Ref. [18], similarly normalized (right axis). The grayscale schematically represents the exponent $\alpha$ in the $T$ dependence of the resistivity $\rho(T) \sim T^{\alpha}$ from Ref. [32]. Here, dark gray, white represents $\alpha=1,2$ respectively.

\section{B. Possible origin of strange metal transport in $\mathrm{FeSe}_{1-x} \mathbf{S}_{x}$}

Overall, $\mathrm{FeSe}_{1-x} \mathrm{~S}_{x}$ exhibits the following signatures of SM transport [22,31-33]: (a) a $T$-linear resistivity above a QC fan extending down to lowest temperatures at or near $x=x_{c}$, (b) correlated FL behavior, i.e., $\rho_{x x}(T) \sim A T^{2}$, below the fan, and (c) a marked enhancement of $A$ upon approaching $x_{c}$ from the high- $x$ side. Since, for reasons of causality, the magnitude of $A$ in most metals scales with $\left(m^{*}\right)^{2}[75,76]$, this implies a tendency for the quasiparticle effective mass $m^{*}$ to diverge at the QCP. In FeSe, $m^{*}$ is already strongly enhanced. The electronic specific heat coefficient of $\mathrm{FeSe}$, due to its two small pockets, is $\sim 50 \%$ higher [41] than band-structure estimates based on contributions from five pockets [49]. Moreover, the mass of the so-called $\gamma$ orbit $\left(m^{*}=7-8 m_{e}[44,49]\right)$ is three to four times larger than the mass of orbits of comparable frequency observed, for example, in underdoped cuprates [77]. For $0 \leqslant x \leqslant x_{c}$, these masses remain high [23], a signature of the dressing of quasiparticles from either nematic and/or magnetic fluctuations. Surprisingly, however, no marked enhancement is observed in $m^{*}$ upon approaching the nematic QCP, neither as a function of $x$ [23] nor pressure [36]. This has led to claims that the nematic QC fluctuations are effectively quenched in $\mathrm{FeSe}_{1-x} \mathrm{~S}_{x}[23,36]$ due to strong nemato-elastic coupling [35] and that the emergent non-FL transport behavior near $x_{c}[18,22,31,33]$ arises instead from spin fluctuations, even though the latter are known to become weaker, not stronger, with increasing $x$ [38].

Such dichotomy in the transport and thermodynamic properties is also found in another electron nematic $\mathrm{Sr}_{3} \mathrm{Ru}_{2} \mathrm{O}_{7}$ [78] (though in contrast to $\mathrm{FeSe}_{1-x} \mathrm{~S}_{x}$ where the nematic phase exists in isolation, the nematic phase in $\mathrm{Sr}_{3} \mathrm{Ru}_{2} \mathrm{O}_{7}$ is found to be intertwined with spin ordering at low $T$ [79]). As the QCP is approached (in an applied magnetic field), $A$ becomes markedly enhanced, yet at the same time, $m^{*}$ of most of the pockets remains unchanged [80]. Above the QCP, a fan of
$T$-linear resistivity then emerges with a coefficient that is consistent with Planckian dissipation [4]. A model explaining the emergence of SM transport in $\mathrm{Sr}_{3} \mathrm{Ru}_{2} \mathrm{O}_{7}$ has recently been proposed by Mousatov et al. [81]. Their model assumes the coexistence of a large FS with a low density of states (DOS), i.e., low $m^{*}$, and a small FS with high DOS caused by a van Hove singularity (vHs) lying just below the Fermi level $E_{F}$. Above a certain temperature (determined by the distance $\epsilon_{h}$ of the vHs from $E_{F}$ and its width $W_{h}$ ), the carriers on the small, heavy FS become nondegenerate, i.e., hot $(h)$. As a result, electrons on the large degenerate (cold) FS are likely to be scattered into these hot spots. Mousatov showed that in such a scenario, the dominant scattering mechanism-denoted as $c c-c h$ scattering - is the one in which two cold (c) electrons collide, one of which is then scattered into the vicinity of the hot spot [81].

In this circumstance, $T$-linear resistivity is realized due to the nondegenerate nature of the hot electrons [81]. Once $k_{B} T<\epsilon_{h}+W_{h}$, electrons at the hot spots become degenerate and the usual $T^{2}$ behavior is restored. The key point of the Mousatov model is that the coefficient of the $T^{2}$ resistivity is governed by the effective mass of the hot electrons $m_{h}^{*}$. Cyclotron motion around the large cold FS, on the other hand, depends only on the light effective mass $m_{c}^{*}$ which does not change on approaching the critical field. In this way, the different behavior of $m^{*}$ extracted from QOs and dc transport can be reconciled. Finally, since $\epsilon_{h} \rightarrow 0$ at the critical field, this same mechanism also gives rise to a QC-like fan of $T$-linear resistivity [81]. Hence, according to the model, coupling to QC fluctuations is not a prerequisite for the appearance of SM transport in $\mathrm{Sr}_{3} \mathrm{Ru}_{2} \mathrm{O}_{7}$.

In FeSe, there is no vHs and thus, at first sight, it is questionable whether the Mousatov model is applicable here. However, striking similarities between the two compounds motivate us to explore an alternative way for the hot spots 
to form in $\mathrm{FeSe}_{1-x} \mathrm{~S}_{x}$ along with the associated mechanism for lifting the electron degeneracy. Indirect evidence for the existence of hot spots in $\mathrm{FeSe}_{1-x} \mathrm{~S}_{x}$ comes from that fact that a quadrature MR of very similar form to that found in $\mathrm{FeSe}_{1-x} \mathrm{~S}_{x}$ has been observed in $\mathrm{BaFe}_{2}\left(\mathrm{As}_{1-x} \mathrm{P}_{x}\right)_{2}$ [14] and recently cited as evidence for hot spot scattering [69]. A recent scanning tunneling spectrosopy study also reported a quasiparticle spectral weight $Z$ in FeSe that was highly anisotropic, with coexisting correlated, marginally coherent and fully coherent quasiparticle states within an individual pocket [64]. Thus, it might be tempting to ascribe the NM and SM components proposed here to these distinct regions in $k$ space; the high DOS tied to the marginally coherent regions forming the hot spots into which cold electrons are scattered, thereby providing the necessary conditions for $c c-c h$ scattering to generate the observed non-FL transport [81].

According to Ref. [81], $\rho_{x x}(T)$ will become $T$ linear only once carriers at the hot spots are nondegenerate, i.e., $k_{B} T>$ $\epsilon_{h}$. For $\mathrm{FeSe}_{1-x} \mathrm{~S}_{x}$, in lieu of the vHs, one might consider using the bottom of the band to set $\epsilon_{h}$. ARPES studies, however, suggest that this scale is around one order of magnitude larger than the temperature scale at which $T$-linear resistivity sets in. Moreover, each pocket grows in size with increasing $x$ [23], implying that $\epsilon_{h}$ would increase rather than decrease upon approaching the QCP. At the same time, however, the chemical pressure created by $\mathrm{S}$ substitution also causes each pocket to become progressively more warped. Ultimately, this may lead to a Lifshitz transition close to $x_{c}$ [23], whereby one of the cylindrical pockets evolves into an ovoid. At the transition, the bottom of the band passes through $\epsilon_{F}$, generating an area of high DOS which would then stay nondegenerate down to the lowest temperatures. Such a DOS sink could then conceivably play the role of the vHs in $\mathrm{FeSe}_{1-x} \mathrm{~S}_{x}$, opening a possible channel for $c c-c h$ scattering and realizing the $T^{2}$ to $T$-linear crossover in $\rho_{x x}(T)$, even if the nematic fluctuations fail to go critical at $x=x_{c}$ and thus to generate Planckian dissipation on their own. Since this Lifshitz transition is claimed to occur close to $x_{c}$ [23] $\left(\epsilon_{h} \rightarrow 0\right)$, the persistence of $T$-linear resistivity down to the lowest temperatures at $x=x_{c}$ can be understood. The presence of this Lifshitz transition, however, is not yet confirmed. Indeed, earlier measurements showed that even in the end member of the series FeS, all pockets remain quasi-two-dimensional $[82,83]$.

Whatever the origin of these putative hot spots, the nematic fluctuations are nonetheless an important element of the overall picture and are most likely the origin of the anisotropic $Z$ found on each pocket [64]. Being $\mathbf{Q}=0$ fluctuations, these fluctuations cannot generate, on their own, sufficient momentum transfer, i.e., large-angle scattering to dominate the dc transport, but in unison with impurity or the residual low-energy spin $[\mathbf{Q}=(\pi, 0)]$ fluctuations [84], they might. Through this process, multiple inelastic $c c-c h$ scattering events, are thus created. The recovery of a $T^{2}$ resistivity in $\rho_{x x}(T)$ beyond $x_{c}$ with a coefficient $A$ that drops markedly with further increase in $x$ indicates a reduction in the quasiparticle-quasiparticle scattering cross section as the system is tuned away from the nematic QCP. Here it is perhaps worth mentioning that the mass determined by QOs is an average of the (extremal) cyclotron orbit that is located furthest from the hot spots and thus may be the least affected by the $c c-c h$ scattering mechanism. Indeed, as already pointed out by Mousatov et al. [81], there must also be a contribution from the usual $c c-c c$ scattering as well, that in the proposed picture would give rise to the NM component. Taking into account the pronounced FS anisotropy in $\mathrm{FeSe}_{1-x} \mathrm{~S}_{x}$, the orbital selectivity and the anisotropy in the scattering rate, the carriers experiencing $c c-c h$ and $c c-c c$ scattering do not have to reside on the same parts of the FS. In light of this, we propose that the Fermi pockets in $\mathrm{FeSe}_{1-x} \mathrm{~S}_{x}$ may be composed of not two, but three distinct regions: those quasiparticles responsible for the NM transport that participate only in the $c c-c c$ scattering, those quasiparticles that generate the SM transport participating only in the $c c-c h$ scattering and those marginally coherent states at the hot spots themselves that give negligible contribution to the transport [81].

From this perspective, it is interesting to return to the discrepancy in the carrier densities extracted from our Hall analysis $\left(=n_{\mathrm{H}}\right)$ and those determined from QO measurements $\left(=n_{\mathrm{QO}}\right)$ [23], as shown in Fig. 5. The observation that $n_{\mathrm{H}} \sim$ $n_{\mathrm{QO}} / 2$ is probably fortuitous, particularly when one takes into account the presence of flat regions or regions of negative FS curvature in the waist of each pocket that might modify the value of $n_{\mathrm{H}}$. Nevertheless, it does suggest that the number of carriers contributing to the NM and SM transport in $\mathrm{FeSe}_{1-x} \mathrm{~S}_{x}$ are comparable. Overall, this analysis suggests that while QOs reflect the total size of the pockets, i.e., the total number of carriers, the NM linear Hall response $\rho_{y x}^{\mathrm{NM}}(H)$, and the corresponding $n_{\mathrm{H}}$ reflect only those parts of the FS that participate in the $c c-c c$ scattering. The rest of the carriers, not visible in the NM Hall response, are assumed to participate in the $c c-c h$ scattering and thereby generate strong signatures of SM transport, including an enhancement in the $A$ coefficient, the $T$-linear resistivity inside the QC fan, the quadrature $\mathrm{MR}$, and the anomalous, nonmonotonic form of $\rho_{y x}^{\mathrm{SM}}(H)$.

Finally, since the effects of a magnetic field have not yet been considered within the Mousatov model, it is not clear how this picture can explain the quadrature MR or $1 / T$ dependence of the SM Hall resistivity (see Sec. VC) seen in $\mathrm{FeSe}_{1-x} \mathrm{~S}_{x}$. Moreover, the effective mixing of $k$ states induced by strong $c h$ scattering may argue against the simple decomposition of the charge response into two independent components as proposed here. Nevertheless, the striking parallels in the evolution of $\rho_{y x}^{\mathrm{SM}}(T, H, x)$ and $\Delta \rho_{x x}^{\mathrm{SM}}(T, H, x)$ shown in Fig. 6, as well as the similarities in the form of $\rho_{y x}^{\mathrm{SM}}(T, H, x)$ found in $\mathrm{FeSe}_{1-x} \mathrm{~S}_{x}$ and in $\mathrm{BaFe}_{2}\left(\mathrm{As}_{1-x} \mathrm{P}_{x}\right)_{2}$ [21], suggest that it is a viable starting point.

\section{Lifetime separation revisited}

In this final section, we return to the issue of the transport anomalies seen at low field, and, in particular, the distinct $T$ dependencies manifest in $\rho_{x x}(T)$ and the inverse Hall angle $\cot \theta_{\mathrm{H}}(T)$ [22]. For FSS16 (and FSS17 [22]), $\rho_{x x}(T)$ is linear or even sublinear below $T=50 \mathrm{~K}$ while $\cot \theta_{\mathrm{H}}(T)=$ $c_{0}+c_{2} T^{2}$. For higher dopings $(0.18 \leqslant x \leqslant 0.22), \rho(T)$ is approximately linear above a certain threshold $T_{1} \leqslant 20 \mathrm{~K}$, yet $\cot \theta_{\mathrm{H}}(T)=c_{0}+c_{2} T^{2}$ up to around $70 \mathrm{~K}$ [see Fig. 7(a)]. As mentioned in the Introduction, this separation of lifetimes is a characteristic of many families of SMs, despite marked differences in FS topology and the distinct nature of the 
(a)

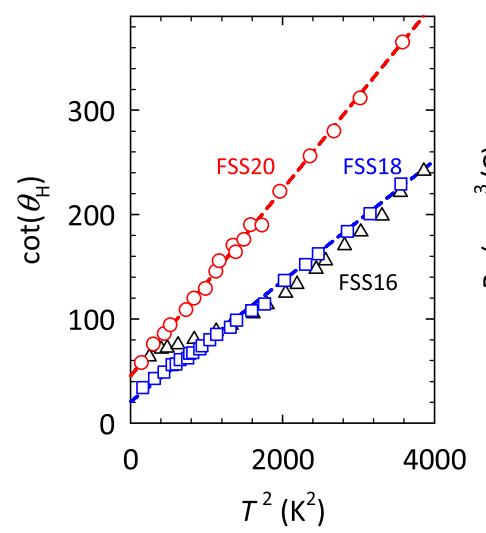

(b)

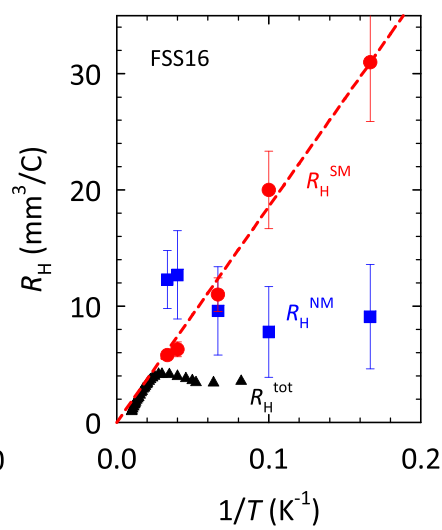

(c)

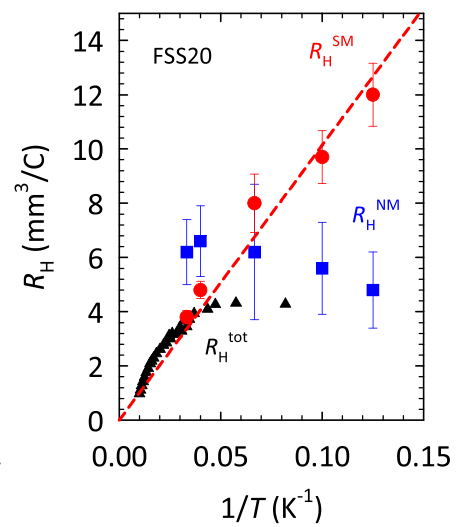

FIG. 7. Deconvolution of the Hall response in $\mathrm{FeSe}_{1-x} \mathrm{~S}_{x}$. (a) $T$ dependence of $\cot \theta_{\mathrm{H}}$ for FSS16 (triangles), FSS18 (squares), and FSS20 (circles). Dashed lines are fits to $\cot \theta_{\mathrm{H}}(T)=c_{0}+c_{2} T^{2}$ (see text). (b), (c) Decomposition of $R_{\mathrm{H}}^{\text {tot }}$ (black triangles) into normal metal $R_{\mathrm{H}}^{\mathrm{NM}}$ (blue squares) and SM $R_{\mathrm{H}}^{\mathrm{SM}}$ (red circles) components for FSS16 and FSS20, respectively. Red dashed lines emphasize the $1 / T$ dependence of $R_{\mathrm{H}}^{\mathrm{SM}}(T)$ (see text).

quantum criticality in each family [85]. The origin of this phenomenon, however, has remained a mystery.

Figures 7(b) and 7(c) show a deconvolution of the low-field $R_{\mathrm{H}}(T)\left[=\rho_{y x}(H) /\left(\mu_{0} H\right)\right]$ plotted versus $1 / T$ for FSS16 and FSS20, respectively. The black symbols in each panel represent the as-measured $R_{\mathrm{H}}(T)$, while the red and blue symbols depict the SM and NM components of $R_{\mathrm{H}}(T)$, respectively. What is striking about these plots is the marked $1 / T$ dependence of $R_{\mathrm{H}}^{\mathrm{SM}}(T)$ extending down to low $T$ but merging with the as-measured $R_{\mathrm{H}}(T)$ at intermediate temperatures. The plateauing of $R_{\mathrm{H}}(T)$ at low- $T$ is then seen to arise through the 'shorting' effect of the (essentially $T$ independent) NM component. This delineation suggests strongly that the strong $T$ dependence of $R_{\mathrm{H}}(T)$ - that is ultimately responsible for the phenomenon of lifetime separation-can be attributed solely to the presence of the SM component, for which $R_{\mathrm{H}}^{\mathrm{SM}}(T)$ has a pure $1 / T$ dependence. Its simple power-law behavior then defines the (low-field) Hall response of the carriers responsible for $T$-linear component of resistivity and quadrature scaling of MR.

\section{CONCLUSIONS}

In summary, detailed analysis of the high-field Hall resistivity in $\mathrm{FeSe}_{1-x} \mathrm{~S}_{x}$ across the nematic QCP reveals that its total Hall response can be decomposed into two contributions: one in which the $\rho_{y x}(H)$ varies linearly with field, the other in which $\rho_{y x}(H)$ shows an anomalous, nonmonotonic response. The linear contribution of the Hall response can be attributed to (compensated) electron and hole quasiparticles. The second component is postulated to derive from hot carriers, presumably located within the same electron and/or hole pockets, that give rise to strange metallic behavior that is most pronounced close to the nematic QCP. The corresponding Hall coefficient of the SM component is found to exhibit a $1 / T$ divergence, which, ultimately, is cut off at low $T$ by the residual NM component. One remaining challenge is to determine whether or not the phenomenology uncovered here can also account for the SM physics observed in other SMs.
The striking similarities in their charge responses certainly motivate further comparison. For $\mathrm{FeSe}_{1-x} \mathrm{~S}_{x}$, a specific challenge remains; to explain how nematic critical fluctuations and spin fluctuations collude to generate such a profound influence on the low- $T \mathrm{dc}$ transport. To this end, magnetotransport measurements on samples with $x>x_{c}$ under applied pressure or strain may prove particularly instructive.

\section{ACKNOWLEDGMENTS}

We thank J. G. Analytis and M. Prosnikov for stimulating discussions. We acknowledge support from the former Foundation for Fundamental Research on Matter (FOM), which is financially supported by the Netherlands Organisation for Scientific Research (NWO) (Grant No. 16METL01) — “Strange Metals" and the European Research Council (ERC) under the European Union's Horizon 2020 research and innovation programme (Grant Agreements No. 835279-Catch-22). This work is supported by the High Field Magnet Laboratory (HFML) at Radboud University (RU), member of the European Magnetic Field Laboratory (EMFL). This work is also supported by Grants-in-Aid for Scientific Research (KAKENHI) (Grants No. JP18H01177, No. JP18H05227, No. JP19H00649, and JP21H04443), Innovative Area "Quantum Liquid Crystals" (Grant No. JP19H05824) from the Japan Society for the Promotion of Science (JSPS), and CREST (No. JPMJCR19T5) from Japan Science and Technology (JST).

\section{APPENDIX A: EVOLUTION OF $T_{s}(x)$ IN $\mathrm{FeSe}_{1-x} \mathrm{~S}_{x}$}

Figure 1(a) shows literature values for $T_{S}(x)$, the onset temperature for the nematic phase in $\mathrm{FeSe}_{1-x} \mathrm{~S}_{x}$, for $x \leqslant 0.25$. Typically, second-order phase transitions lead to a jump in the specific heat $\Delta C$; the (mean-field) transition temperature coinciding with the midpoint of $\Delta C(T)$ on the high-temperature side. In $\mathrm{FeSe}_{1-x} \mathrm{~S}_{x}, T_{s}(x)$ is frequently determined using the minimum in the $T$ derivative of the in-plane resistivity $d \rho_{x x} / d T$ at $T=T_{\min }$ or by the midpoint of the step. This can lead to an underestimate of $T_{s}(x)$ in $\mathrm{FeSe}_{1-x} \mathrm{~S}_{x}$ by as 
(a)

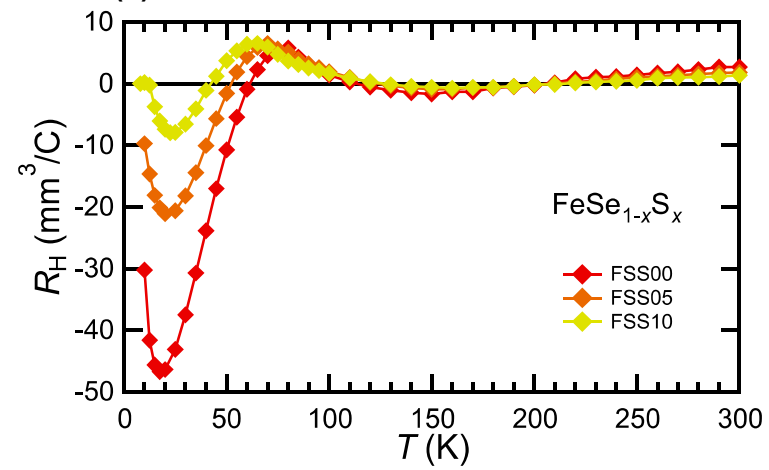

(b)

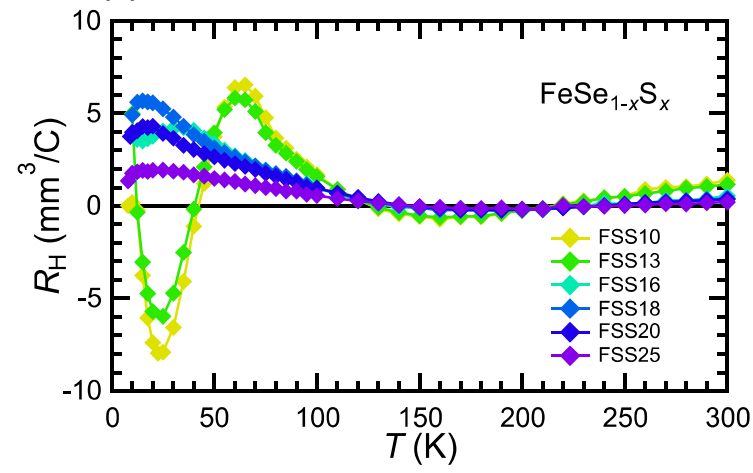

FIG. 8. Low-field $\left(\mu_{0} H=1 \mathrm{~T}\right)$ Hall coefficient $R_{\mathrm{H}}$ in $\mathrm{FeSe}_{1-x} \mathrm{~S}_{x}$ as a function of $x$ and $T$. The data are shown in two panels for clarity.

much as $10-15 \mathrm{~K}$. For FeSe, $T_{s}$ actually coincides with the shoulder, i.e., the onset of the steplike feature in $d \rho_{x x} / d T$ at $T=T_{\mathrm{sh}}$, as found in other systems [86]. For this reason, the data points shown in Fig. 1(a) are taken from the location of $T_{\mathrm{sh}}(x)$. Despite using such a unifying definition, there is still a spread in values, reflecting both uncertainties in $x$ and the sensitivity of $T_{s}$ to disorder. Typically, the $\mathrm{S}$ content in $\mathrm{FeSe}_{1-x} \mathrm{~S}_{x}$ is determined by energy dispersive X-ray (EDX) spectroscopy. Where quoted, EDX has an uncertainty in $x$ of \pm 0.01 within an individual crystal [42] and $\sim \pm 0.02$ within a single batch of crystals [38]. A detailed disorder study in FeSe meanwhile found that $T_{s}$ can drop by up to $20 \mathrm{~K}$ in samples with a low residual resistivity ratio [87].

As shown in Fig. 1(a), our values for $T_{s}(x)$ tend to lie above the average. Early studies suggested that the nominal value of $x$, as used here, was higher than the actual $x$ content (as determined by EDX) by as much as $20 \%$ [28]. We note that this is comparable to the absolute uncertainty in EDX. Nevertheless, it is indeed possible that the quoted $x$ values for FSS10, FSS13, and FSS16 are an overestimate. For all other samples, however, both their resistivity and (low-field) Hall responses are identical to those with similar (but nonetheless EDX determined) $x$ values reported elsewhere $[22,23]$. Importantly, this places all higher S-content samples, including FSS18, above the critical concentration $x_{c}=0.17$. Finally, a study by Böhmer et al. [87] highlighted how increased disorder not only shifts $T_{s}$ to lower $T$ but also broadens the associated resistive transition. In our crystals, we observe sharp transitions with $T_{\mathrm{sh}}-T_{\min } \leqslant 5 \mathrm{~K}$ for all $x$ [bar FSS16 where the gradient in $T_{s}(x)$ is the steepest]. These values are approximately half those typically reported $[23,31,38,42]$. Hence, it is also possible that our $T_{s}(x)$ values are elevated with respect to others due to reduced levels of disorder.

\section{APPENDIX B: LOW-FIELD HALL EFFECT MEASUREMENTS}

Figure 8 shows $R_{\mathrm{H}}(T)$ in $\mathrm{FeSe}_{1-x} \mathrm{~S}_{x}$ between $10 \mathrm{~K}$ and $300 \mathrm{~K}$ for $\mu_{0} H=1 \mathrm{~T}$ for the same series of single crystals used in the high-field measurements plus two additional samples: $x=0.05$ and $x=0.18$. As can be seen, $R_{\mathrm{H}}$ exhibits a complex $T$ dependence for all $x$, changing sign twice for $x \geqslant 0.16$ and three or even four times for $x<0.16$, reflecting their multiband nature. The overall evolution of the low-field
Hall effect in our crystals is consistent with that reported previously [22]. There is a clear difference in the behavior of the low- $T R_{\mathrm{H}}$ across the nematic QCP. Moreover, examination of the field dependence of $R_{\mathrm{H}}(T)$, shown in Fig. 9, reveals a clear sign change in the field dependence beyond $x_{c} \sim 0.17$. Overall, the nonlinearity softens with both increasing $x$ and increasing $T$.

\section{APPENDIX C: APPLICATION OF TWO- AND THREE-BAND MODELS}

The high-field data shown in Fig. 1(b) of the main text illustrate the general trends in the evolution of the Hall response of $\mathrm{FeSe}_{1-x} \mathrm{~S}_{x}$ with $x$ and with magnetic field. Our previous MR study [18] showed that for the same series of samples, the longitudinal MR does not follow a simple $H^{2}$ dependence. Such behavior is usually ascribed to the presence of multiple pockets of opposite polarity. $\mathrm{FeSe}_{1-x} \mathrm{~S}_{x}$ is known to contain a number of bands crossing the Fermi level, though the precise number of pockets has been the subject of debate. Recently, however, a consensus has begun to emerge, at least for FeSe below $T_{s}$, where a single pair of compensated electron and hole pockets survive. In S-substituted FeSe, up to five individual QO frequencies have been observed [23], implying that an additional pocket may appear at finite $x$. Hence, in the following, we investigate whether standard two- or three-band models can self-consistently capture the combined Hall and MR data of $\mathrm{FeSe}_{1-x} \mathrm{~S}_{x}$. Our conclusion is that both models fail, indicating the need for either more complicated modeling or an interpretation beyond standard Drude.

\section{Two-band model}

According to Boltzmann transport theory, only a perfectly isotropic single band system shows a $H$-linear Hall resistivity, $\rho_{y x}=R_{\mathrm{H}} \times \mu_{0} H$, with $R_{\mathrm{H}}=1 /(n e)$. The corresponding MR in such a system is zero. One of the simplest systems to exhibit a nonlinear $\rho_{y x}(H)$ and a finite MR is a two-band system composed of one electron and one hole pocket, both of which are isotropic. In this case, the longitudinal and Hall resistivities are given by [88]

$$
\rho_{x x}(H)=\frac{1}{e} \frac{\left(n_{h} \mu_{h}+n_{e} \mu_{e}\right)+\left(n_{h} \mu_{e}+n_{e} \mu_{h}\right) \mu_{h} \mu_{e}\left(\mu_{0} H\right)^{2}}{\left(n_{h} \mu_{h}+n_{e} \mu_{e}\right)^{2}+\left(n_{h}-n_{e}\right)^{2} \mu_{h}^{2} \mu_{e}^{2}\left(\mu_{0} H\right)^{2}},
$$


(a)

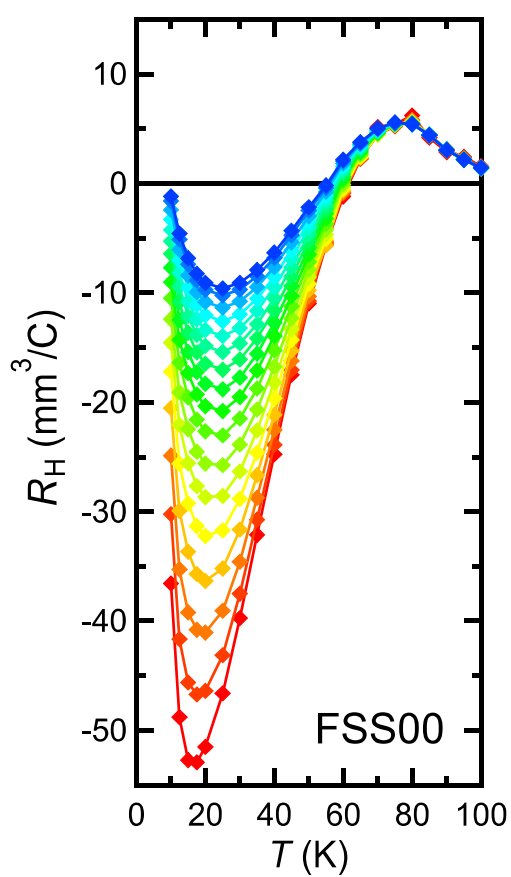

(e)

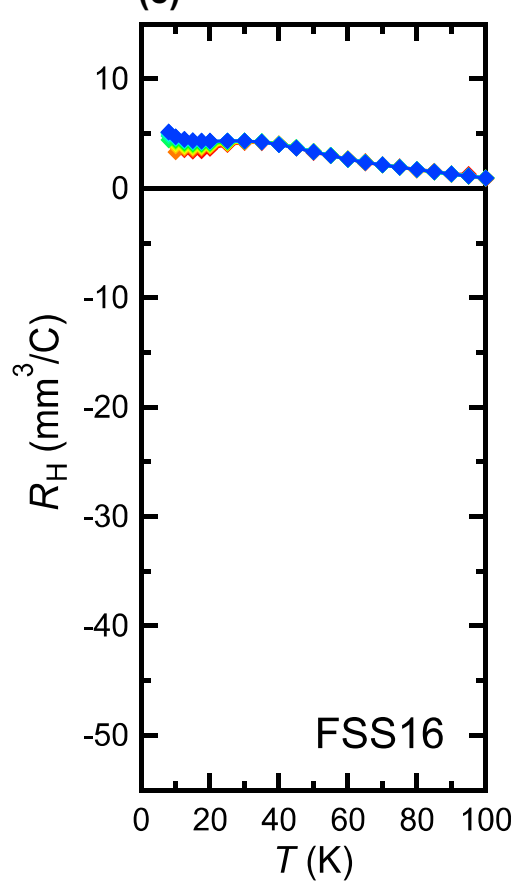

(b)

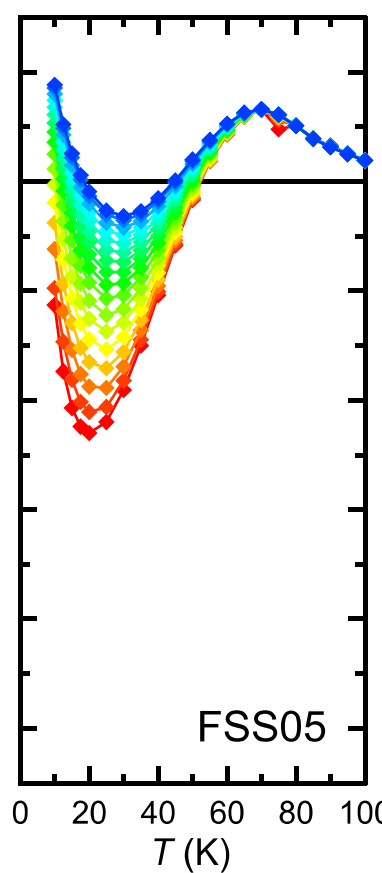

(f)

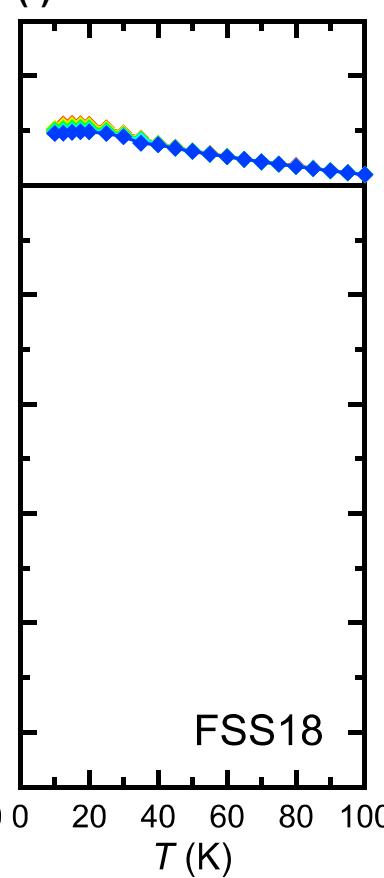

(c)

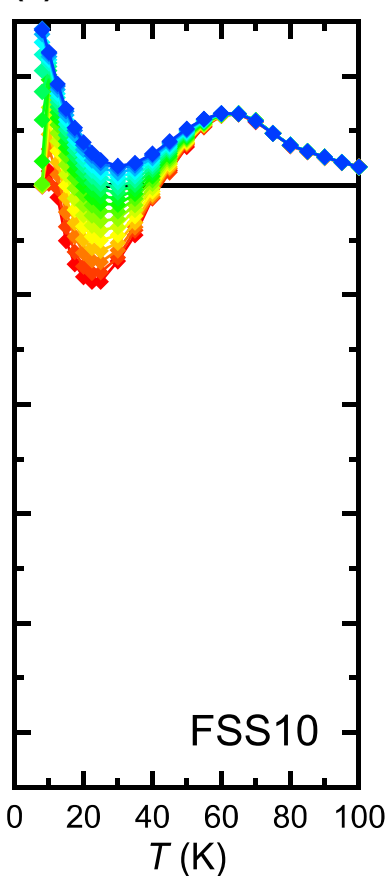

(g)

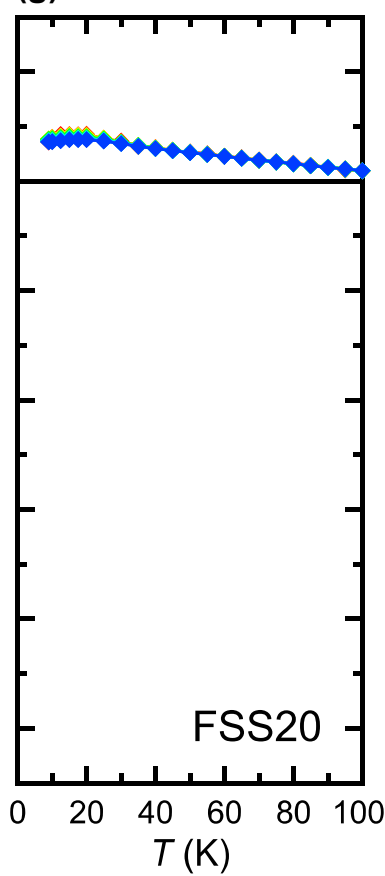

(d)

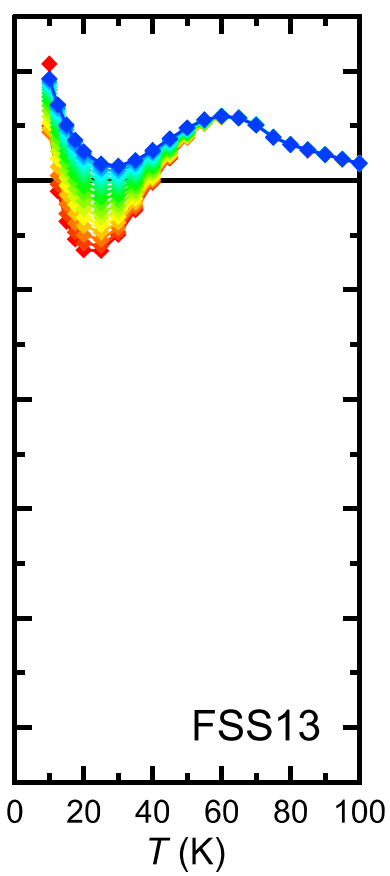

(h)

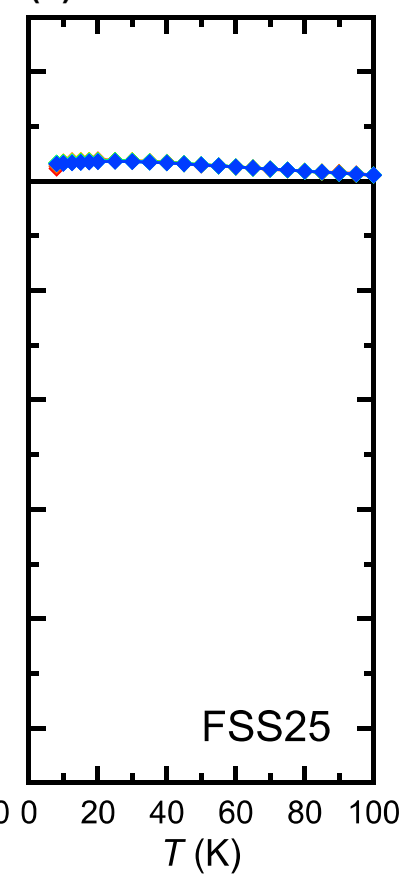

FIG. 9. Temperature dependence of the low-field Hall coefficient $R_{\mathrm{H}}$ in $\mathrm{FeSe}_{1-x} \mathrm{~S}_{x}$ at different magnetic field strengths between $0.5 \mathrm{~T}$ (red) and $8 \mathrm{~T}$ (blue) in incremental steps of $0.5 \mathrm{~T}$. The data are shown for (a) FSS00, (b) FSS05, (c) FSS10, (d) FSS13, (e) FSS16, (f) FSS18, (g) FSS20, and (h) FSS25. Note the field dependence changes sign above $x_{c}=0.17$.

$\rho_{y x}(H)=\frac{\mu_{0} H}{e} \frac{\left(n_{h} \mu_{h}^{2}+n_{e} \mu_{e}^{2}\right)+\left(n_{h}-n_{e}\right) \mu_{h}^{2} \mu_{e}^{2}\left(\mu_{0} H\right)^{2}}{\left(n_{h} \mu_{h}+n_{e} \mu_{e}\right)^{2}+\left(n_{h}-n_{e}\right)^{2} \mu_{h}^{2} \mu_{e}^{2}\left(\mu_{0} H\right)^{2}}$,

where $n_{e}$ is the density of electrons, $n_{h}$ the hole density, $\mu_{e}$ the electron mobility, and $\mu_{h}$ the hole mobility. Note that for a perfectly compensated two-band system $\left(n_{e}=n_{h}\right), \rho_{x x}(H) \sim$ $H^{2}$ and $\rho_{y x}(H) \sim H$. Therefore, to account for the deviations from these simple relations in $\mathrm{FeSe}_{1-x} \mathrm{~S}_{x}$, we must first relax the condition of perfect compensation.

A simple rearrangement of Eqs. (C1) and (C2) gives the expressions

$$
\begin{aligned}
& \frac{\rho_{x x}(x)}{\rho_{x x}(0)}=\frac{1+a x^{2}}{1+b x^{2}}, \\
& \rho_{y x}(x)=\frac{c+d x^{2}}{1+b x^{2}} x,
\end{aligned}
$$



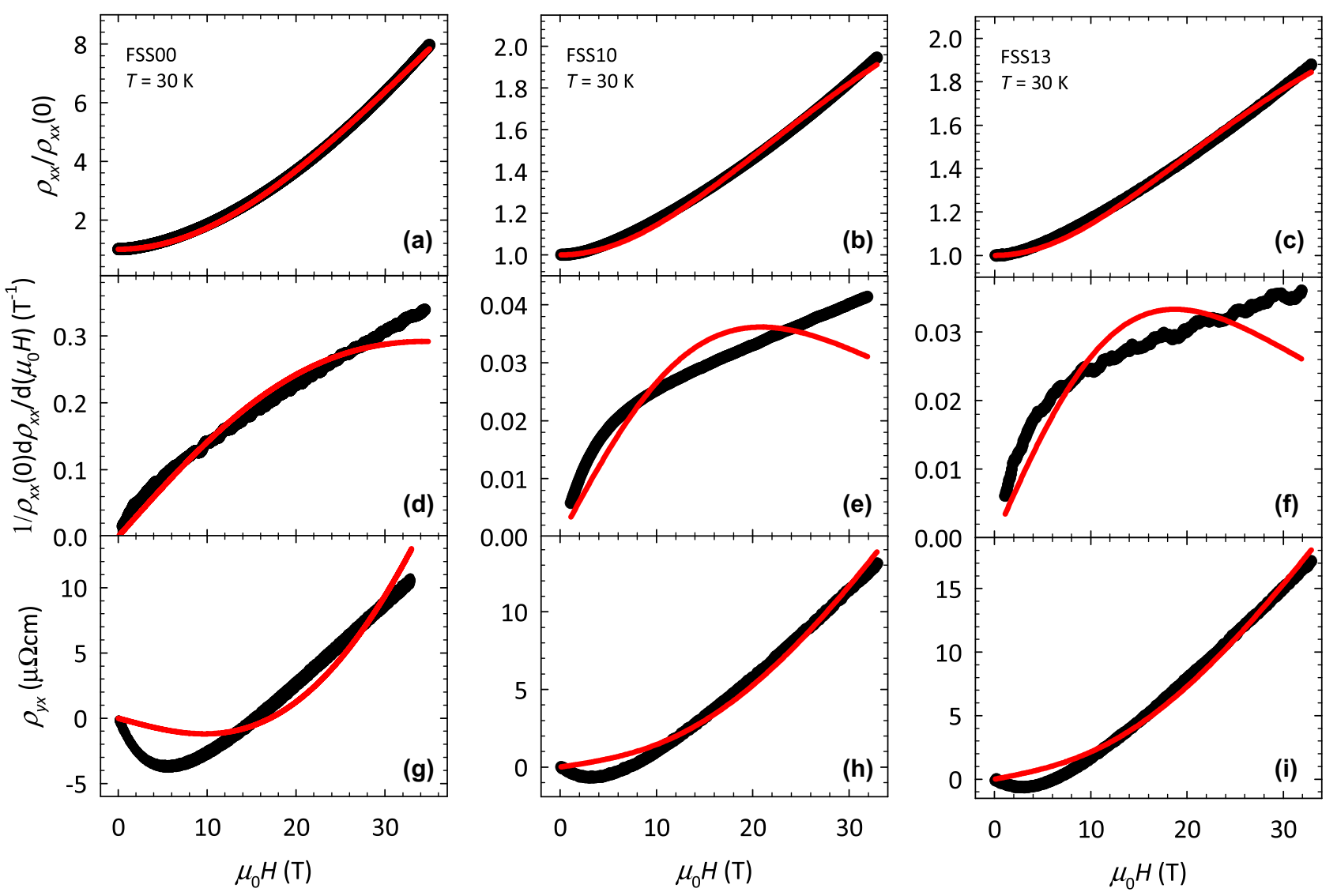

FIG. 10. Application of the two-band model to the magnetotransport of $\mathrm{FeSe}_{1-x} \mathrm{~S}_{x}$. (a)-(c) Fitting of the longitudinal resistivity $\rho_{x x}(H)$ normalized to the zero-field value $\rho_{x x}(0)$ [18] using Eq. (C3). The fits are shown for (a) FSS00, (b) FSS10, and (c) FSS13 at $T=30 \mathrm{~K}$. (d)-(f) Fitting of the field derivative $1 / \rho_{x x}(0)\left(d \rho_{x x} / d H\right)$. Black lines represent the measured data and red lines the fits to Eq. (C3). (g)-(i) Simultaneous fitting of the Hall resistivity $\rho_{y x}(H)$ using Eq. (C4) with fitting parameter $b$ taken from the fit to the MR data (see text). Again, black lines represent the measured data and red lines the fits to Eq. (C4).

where $\quad x=\mu_{0} H, \quad \rho_{x x}(0)=1 /\left(e n_{e} \mu_{e}+e n_{h} \mu_{h}\right), \quad a=$ $e \rho_{x x}(0)\left(n_{h} \mu_{e}+n_{e} \mu_{h}\right) \mu_{h} \mu_{e}, \quad b=e^{2} \rho_{x x}^{2}(0)\left(n_{h}-n_{e}\right)^{2} \mu_{h}^{2} \mu_{e}^{2}$, $c=e \rho_{x x}^{2}(0)\left(n_{h} \mu_{h}^{2}-n_{e} \mu_{e}^{2}\right)$, and $d=e \rho_{x x}^{2}(0)\left(n_{h}-n_{e}\right) \mu_{h}^{2} \mu_{e}^{2}$; $a, b, c$, and $d$ are thus the relevant fitting parameters, though, normally, only three of these are completely independent. Nevertheless, in the following, we will assume that all fitting parameters are free. To check the applicability of the two-band model, we must fit both $\rho_{y x}(H)$ and $\rho_{x x}(H)$ in a self-consistent way. Note that $\rho_{x x}(H)$ and $\rho_{y x}(H)$ share a common denominator which strongly constrains the fitting. There are multiple ways of performing this fitting routine self-consistently. Here we decided to first perform a fit of the previous MR data [18] using Eq. (C3) without constraining either $a$ or $b$. After that, we fit the $\rho_{y x}(H)$ data using Eq. (C4) with the same value of $b$. The results of this fitting procedure are shown in Fig. 10 for FSS00, FSS10, and FSS13.

A quick glance of the top panels in Fig. 10 suggests that the two-band model actually gives a reasonable fit to $\rho_{x x}(H)$. However, as shown in the middle panels, a closer inspection of the field derivative of $\rho_{x x}(H)$, shows clearly that the tendency of the fitting curve toward saturation at higher fields is never realized in the actual data. Indeed, $1 / \rho_{x x}(0)\left(d \rho_{x x} / d H\right)$ invariably has both a finite intercept and a constant slope, indicating that the high-field MR exhibits a parabolic field dependence, i.e., $\rho_{x x}(H)=\rho_{x x}(0)+a_{1} H+a_{2} H^{2}$. Clearly, the unconstrained fits to Eq. (C3) cannot replicate such behavior. The failure of the two-band model is also more evident in the constrained fits of $\rho_{y x}(H)$ to Eq. (C4) as shown in the lower panels of Fig. 10, which completely fail to reproduce the essential features of the data. We thus conclude that the two-band model alone cannot describe the magnetotransport properties of the nematic phase in $\mathrm{FeSe}_{1-x} \mathrm{~S}_{x}$.

\section{Three-band model}

Explicit formulas for the transport coefficients of an arbitrary multiband system have been derived by Kim using a matrix formalism [56]. In the case of a three-band system, the appropriate expressions are

$$
\begin{aligned}
& \frac{\rho_{x x}(x)}{\rho_{x x}(0)}=\frac{1+a x^{2}+b x^{4}}{1+c x^{2}+d x^{4}}, \\
& \rho_{y x}(x)=\frac{e+f x^{2}+g x^{4}}{1+c x^{2}+d x^{4}} x,
\end{aligned}
$$

where again $x=\mu_{0} H$ and $a, b, c, d, e, f$, and $g$ are the fitting parameters which depend on individual carrier densities and mobilities. Although normally only five parameters are completely independent, we will assume that all parameters are free. Here, $\rho_{x x}(H)$ and $\rho_{y x}(H)$ share two common fitting parameters, $c$ and $d$, in their denominators, though in a perfectly 

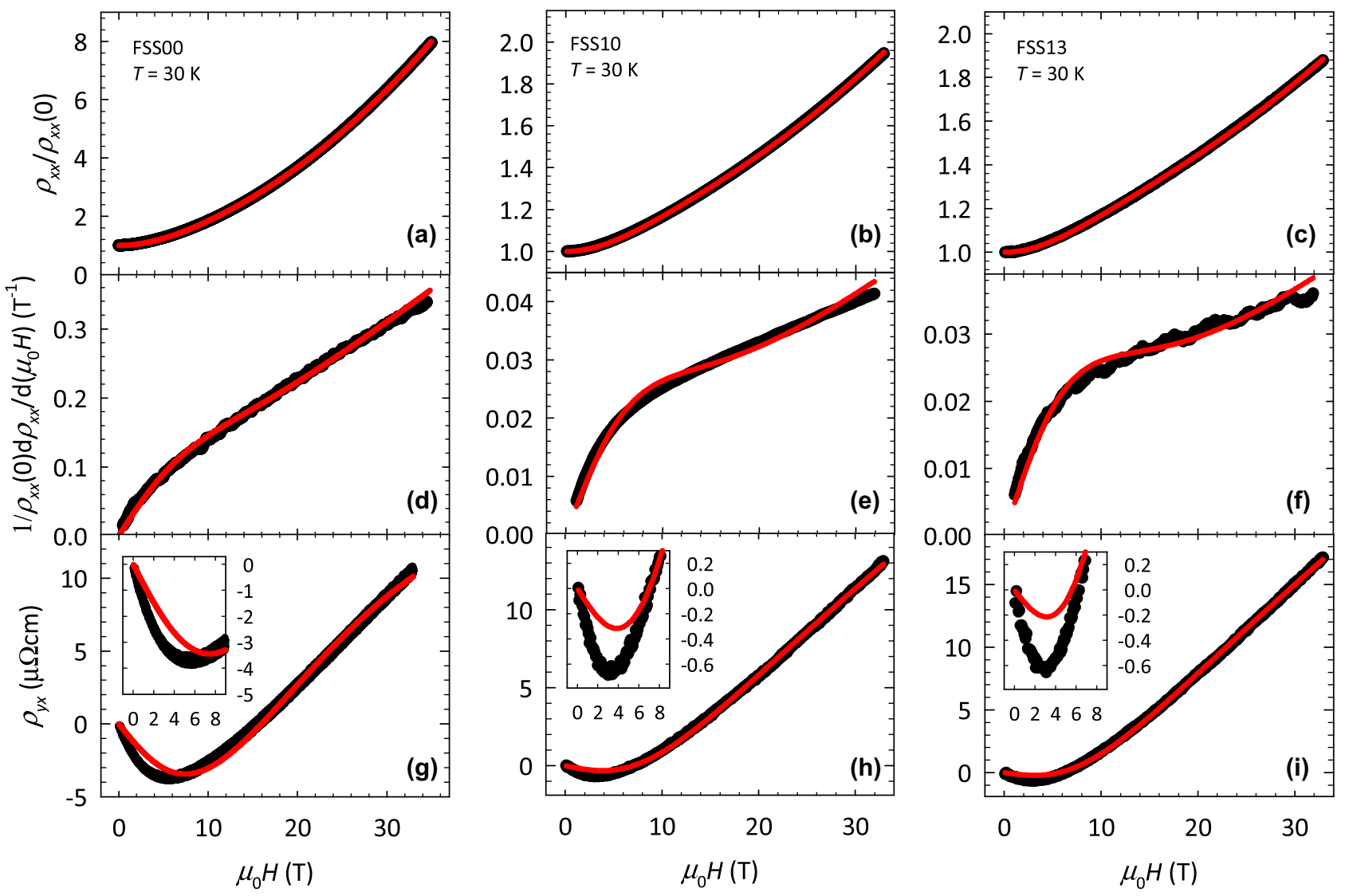

FIG. 11. Application of the compensated three-band model to the magnetotransport of $\mathrm{FeSe}_{1-x} \mathrm{~S}_{x}$. (a)-(c) Fitting of $\rho_{x x}(H) / \rho_{x x}(0)$ [18] using Eq. (C5) with the fitting parameter $d=0$. The fits are shown for (a) FSS00, (b) FSS10, and (c) FSS13 at 30 K. (d)-(f) Fitting to the field-derivative $1 / \rho_{x x}(0)\left(d \rho_{x x} / d H\right)$ for the same data set. Black lines represent the measured data and red lines the fits to Eq. (C5). (g)-(i) Simultaneous fitting of $\rho_{y x}(H)$ using Eq. (C6) with $d=0$ and $c$ taken from the fit to the MR data. Black lines represent the measured data and red lines the fits to Eq. (C6). Insets: Enlargement of the low-field part.

compensated system, $d=0$ [56]. This model was previously applied to successfully explain the low-field magnetotransport of pure FeSe [44] and $\mathrm{BaFe}_{2} \mathrm{As}_{2}$ [58]. In both cases, perfect compensation was assumed.

As with the two-band model, we first perform the fit to the $\rho_{x x}(H)$ data using Eq. (C5) letting all parameters (bar $d=0$ ) be free. After that, we fit the $\rho_{y x}(H)$ data using Eq. (C6), again with $d=0$ and $c$ set equal to its value found from the MR fit. The results of this fitting procedure are shown in Fig. 11 for the same three samples FSS00, FSS10, and FSS13.

The fits to the compensated three-band model are of course better than those for the two-band model. Nevertheless, inspection of the field derivatives (middle panels of Fig. 11) reveals additional features in the fits that are absent in the data. Moreover, the constrained fits to $\rho_{y x}(H)$ (bottom panels) in Fig. 11 also fail to capture the low-field response, as highlighted in the insets.

To proceed further, we relax the constraint of perfect compensation (i.e., we allow $d$ to be finite) and perform simultaneous fits to $\rho_{y x}(H)$ and $\rho_{x x}(H)$ using Eqs. (C5) and (C6). The results are shown in Fig. 12. As expected, the fits to the uncompensated three-band model are again improved, particularly for $\rho_{x x}(H)$. Nevertheless, even with this wholly unconstrained fitting procedure, we struggle to capture the low-field feature in $\rho_{y x}(H)$, espe- cially for FSS10 and FSS13 [see insets of Figs. 12(g) and 12(h)].

Of course, one could always incorporate additional pockets into the model or add further complexity into the two- or three-band models, such as field-dependent mobilities and carrier densities, band anisotropies, etc. Importantly, however, the very specific form of the MR in $\mathrm{FeSe}_{1-x} \mathrm{~S}_{x}$ (particularly in more disordered samples [18] where the fitting is expected to be much simpler), coupled with the marked changes in the Hall response as a function of $x$, are incompatible with any standard multiband model. It is for this reason that we proceed to consider the alternative scenario outlined in the main text that incorporates both NM and SM components.

\section{APPENDIX D: PARALLEL CONDUCTIVITY CHANNEL APPROXIMATION}

Within a parallel conductivity picture, the total conductivity $\sigma_{x x}^{\text {tot }}$ of the system can be written as

$$
\sigma_{x x}^{\mathrm{tot}}=\sigma_{x x}^{\mathrm{NM}}+\sigma_{x x}^{\mathrm{SM}}
$$

where $\sigma_{x x}^{\mathrm{NM}}$ and $\sigma_{x x}^{\mathrm{SM}}$ are the normal and SM contributions to $\sigma_{x x}^{\text {tot }}$, respectively. The transverse magnetoconductance is then 

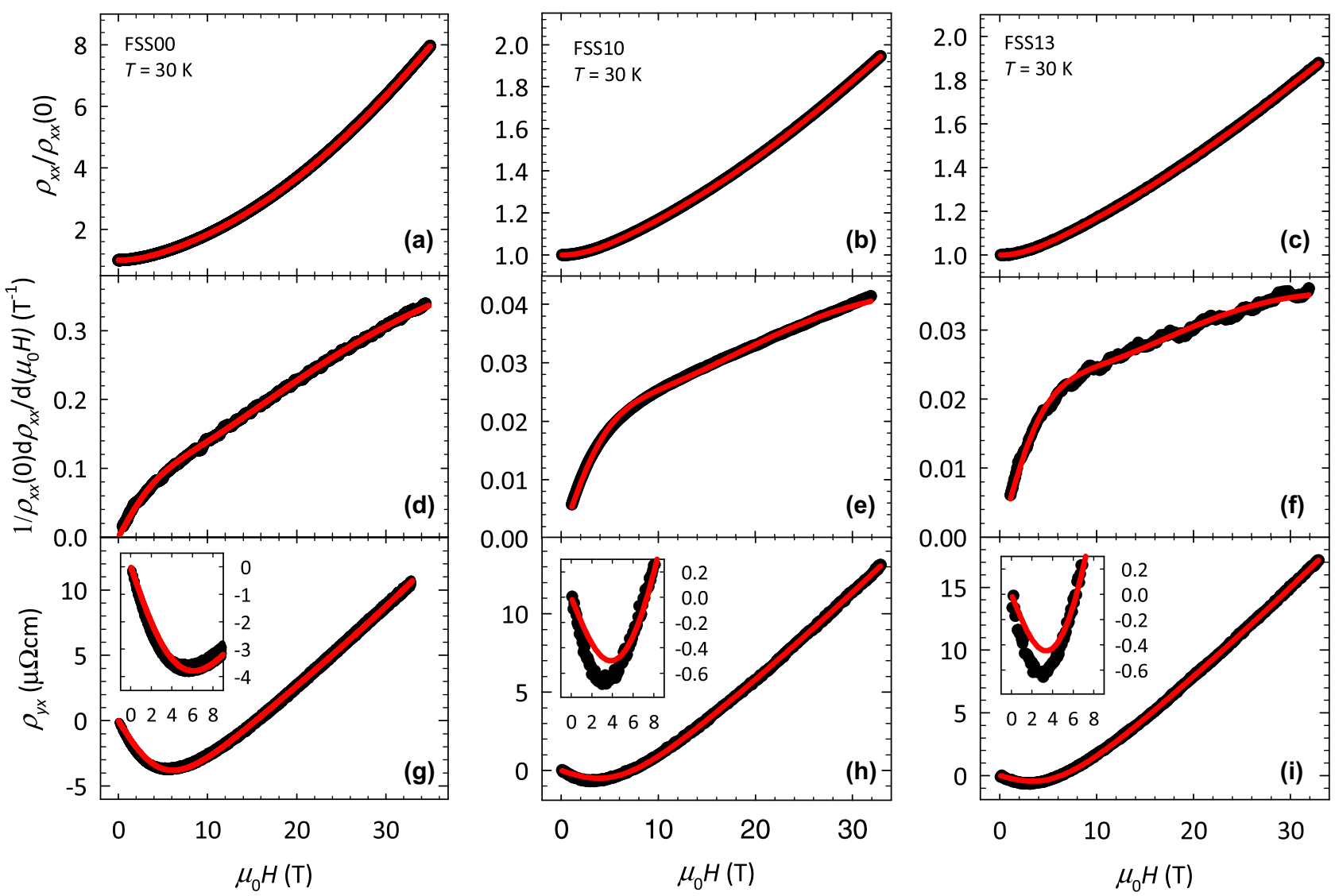

FIG. 12. Application of the uncompensated three-band model to the magnetotransport in $\mathrm{FeSe}_{1-x} \mathrm{~S}_{x}$. (a)-(c) Fitting of $\rho_{x x}(H) / \rho_{x x}(0)$ [18] using Eq. (C5) without any constraints. The fits are shown for (a) FSS00, (b) FSS10, and (c) FSS13 at 30 K. (d)-(f) Fits to the field-derivative $1 / \rho_{x x}(0)\left(d \rho_{x x} / d H\right)$. Black lines represent the measured data and red lines the fits to Eq. (C5). (g)-(i) Simultaneous fitting of $\rho_{y x}(H)$ using Eq. (C6) with the fitting parameters $c$ and $d$ taken from the fit to the MR data (see text). Again, black lines represent the measured data and red lines the fits to Eq. (C6). Insets: Enlargement of the low-field part.

given by the weighted sum:

$$
\frac{\Delta \sigma_{x x}^{\mathrm{tot}}}{\sigma_{x x}^{\mathrm{tot}}(H)}=\frac{\sigma_{x x}^{\mathrm{NM}}(H)}{\sigma_{x x}^{\text {tot }}(H)} \frac{\Delta \sigma_{\mathrm{NM}}}{\sigma_{x x}^{\mathrm{NM}}(H)}+\frac{\sigma_{x x}^{\mathrm{SM}}(H)}{\sigma_{x x}^{\text {tot }}(H)} \frac{\Delta \sigma_{\mathrm{SM}}}{\sigma_{x x}^{\mathrm{SM}}(H)}
$$

where $\Delta \sigma_{x x}(H)=\sigma_{x x}(H)-\sigma_{x x}(0)$. In reality, it is the MR $\left[\Delta \rho_{x x}(H)=\rho_{x x}(H)-\rho_{x x}(0)\right]$, rather than the magnetoconductance that is measured, the former being related to the latter via inversion of the (in-plane) conductivity tensor:

$$
\frac{\Delta \rho_{x x}}{\rho_{x x}(0)}=-\frac{\sigma_{x x}(H) \Delta \sigma_{x x}}{\sigma_{x x}^{2}(H)+\sigma_{y x}^{2}(H)}-\frac{\sigma_{y x}^{2}(H)}{\sigma_{x x}^{2}(H)+\sigma_{y x}^{2}(H)} .
$$

To proceed, we make a number of approximations. Our first is the usual assumption that $\sigma_{y x}^{2}(H) \ll \sigma_{x x}^{2}(H)$, leading to

$$
\frac{\Delta \rho_{x x}}{\rho_{x x}(0)} \approx-\frac{\Delta \sigma_{x x}}{\sigma_{x x}(H)}-\frac{\sigma_{y x}^{2}(H)}{\sigma_{x x}^{2}(H)} .
$$

The second approximation is to assume that the MR is dominated by the magnetoconductance term $\Delta \sigma_{x x} / \sigma_{x x}(H)$, i.e., that

$$
\frac{\Delta \sigma_{x x}}{\sigma_{x x}(H)} \gg \frac{\sigma_{y x}^{2}(H)}{\sigma_{x x}^{2}(H)},
$$

after which we obtain

$$
\frac{\Delta \rho_{x x}(H)}{\rho_{x x}(0)} \approx-\frac{\Delta \sigma_{x x}(H)}{\sigma_{x x}(H)} .
$$

Putting Eq. (D6) into Eq. (D2), we get

$$
\begin{aligned}
\Delta \rho_{x x}^{\text {tot }} \approx & \left(\frac{\sigma_{x x}^{\mathrm{NM}}(0)}{\sigma_{x x}^{\text {tot }}(0)}\right)\left(\frac{\sigma_{x x}^{\mathrm{NM}}(H)}{\sigma_{x x}^{\text {tot }}(H)}\right) \Delta \rho_{\mathrm{NM}} \\
& +\left(\frac{\sigma_{x x}^{\mathrm{SM}}(0)}{\sigma_{x x}^{\text {tot }}(0)}\right)\left(\frac{\sigma_{x x}^{\mathrm{SM}}(H)}{\sigma_{x x}^{\text {tot }}(H)}\right) \Delta \rho_{\mathrm{SM}} .
\end{aligned}
$$

Our final approximation ignores the field dependence of the ratio of the NM/SM conductivity component and the total conductivity:

$$
\begin{aligned}
& \frac{\sigma_{x x}^{\mathrm{NM}}(H)}{\sigma_{x x}^{\text {tot }}(H)} \approx \frac{\sigma_{x x}^{\mathrm{NM}}(0)}{\sigma_{x x}^{\text {tot }}(0)}, \\
& \frac{\sigma_{x x}^{\mathrm{SM}}(H)}{\sigma_{x x}^{\text {tot }}(H)} \approx \frac{\sigma_{x x}^{\mathrm{SM}}(0)}{\sigma_{x x}^{\text {tot }}(0)} .
\end{aligned}
$$

This gives, finally,

$$
\Delta \rho_{x x}^{\text {tot }} \approx\left(\frac{\sigma_{x x}^{\mathrm{NM}}(0)}{\sigma_{x x}^{\text {tot }}(0)}\right)^{2} \Delta \rho_{x x}^{\mathrm{NM}}+\left(\frac{\sigma_{x x}^{\mathrm{SM}}(0)}{\sigma_{x x}^{\text {tot }}(0)}\right)^{2} \Delta \rho_{x x}^{\mathrm{SM}},
$$


(a)

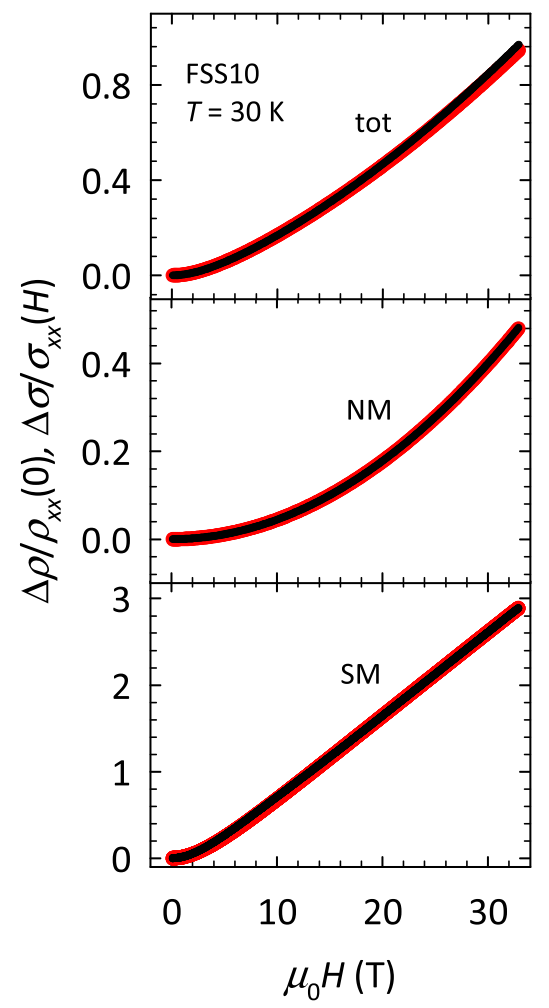

(b)

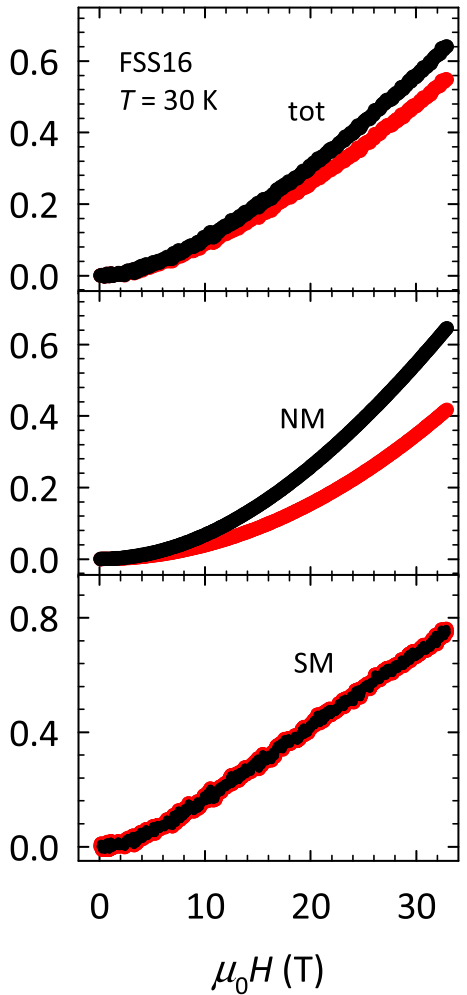

(c)

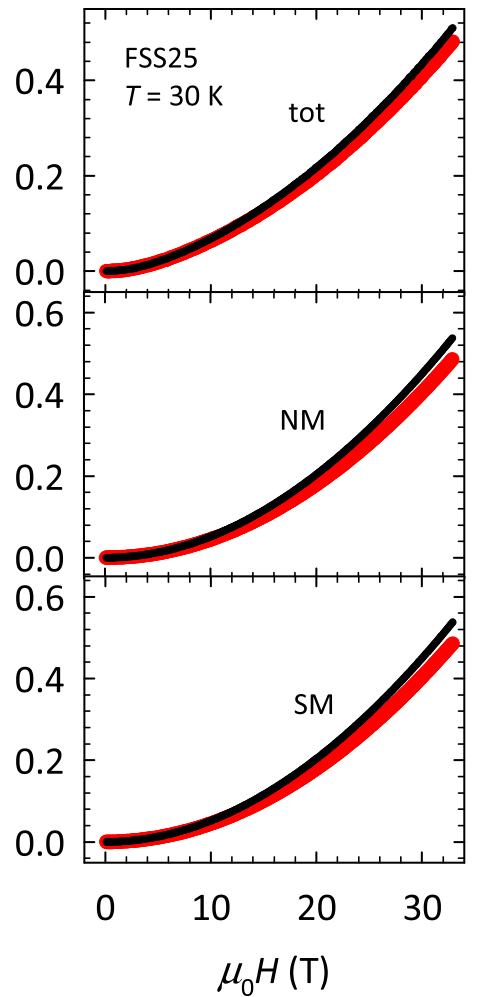

FIG. 13. Test of the validity of approximation (D6). $\Delta \rho_{x x}(H) / \rho_{x x}(0)$ and $\Delta \sigma_{x x}(H) / \sigma_{x x}(H)$ at $30 \mathrm{~K}$ for (a) FSS10, (b) FSS16, and (c) FSS25. Top panels refer to the total measured quantities, middle panels to the NM component, and the bottom panels to the SM component. Red lines represent $\Delta \rho_{x x}(H) / \rho_{x x}(0)$ and black lines $\Delta \sigma_{x x}(H) / \sigma_{x x}(H)$.

which is the relation used in our previous MR study [18]. Equation (D9) states that in the parallel conductivity model, the total measured MR can be approximated as a weighted sum of the MR contributions coming from the separate channels with the weighting factors $\left[\sigma_{x x}^{\mathrm{NM}}(0) / \sigma_{x x}^{\text {tot }}(0)\right]^{2}$ and $\left[\sigma_{x x}^{\mathrm{SM}}(0) / \sigma_{x x}^{\text {tot }}(0)\right]^{2}$ being a measure of the contribution of each respective channel to the total zero-field conductivity.

Before proceeding, we first examine the validity of the three approximations employed. Figure 13 shows a comparison of $\Delta \rho_{x x}(H) / \rho_{x x}(0)$ and $\Delta \sigma_{x x}(H) / \sigma_{x x}(H)$ as a function of magnetic field for the total, NM and SM contributions for three representative concentrations, FSS10, FSS16, and FSS25 at $30 \mathrm{~K}$. (Similar values were obtained at all measured temperatures and also for FSS13 and FSS20). As demonstrated in Fig. 13, Eq. (D6) holds reasonably well for all three quantities at all concentrations. Only in case of the NM component for FSS16 is the difference between $\Delta \rho_{x x}(H) / \rho_{x x}(0)$ and $\Delta \sigma_{x x}(H) / \sigma_{x x}(H)$ somewhat bigger, but even there the maximum error introduced is never more than $35 \%$.

Figure 14 shows the field dependence of $\sigma_{x x}^{\mathrm{NM}} / \sigma_{x x}^{\text {tot }}$ and $\sigma_{x x}^{\mathrm{SM}} / \sigma_{x x}^{\text {tot }}$ for the same concentrations at $30 \mathrm{~K}$. According to Eq. (D8), $\sigma_{x x}^{\mathrm{NM}} / \sigma_{x x}^{\text {tot }}$ and $\sigma_{x x}^{\mathrm{SM}} / \sigma_{x x}^{\text {tot }}$ should not exhibit significant field dependence. As can be seen in Fig. 14, this statement holds very well for FSS16 and FSS25 at $30 \mathrm{~K}$ (and at all other temperatures studied) and also for FSS20 (not shown). For FSS10 (and FSS13), however, $\sigma_{x x}^{\mathrm{NM}} / \sigma_{x x}^{\text {tot }}$ and $\sigma_{x x}^{\mathrm{SM}} / \sigma_{x x}^{\text {tot }}$ exhibits a pronounced field dependence. This is a consequence of the MR which increases with decreasing $x$, reaching $100 \%$ at $30 \mathrm{~K}$ (see Fig. 10). As it turned out, analysis of the Hall effect for FSS10 and FSS13 proved more challenging than for the other concentrations as will be discussed in the following section.

\section{APPENDIX E: SEARCH FOR THE HALL RESPONSE OF THE STRANGE METAL COMPONENT}

Assuming parallel conduction, the diagonal element of the total conductivity tensor $\sigma_{x x}^{\text {tot }}$ is given by Eq. (D1) and the MR for the combined components is then approximated by the Eq. (D9), i.e., by a weighted sum of two independent contributions. In a similar vein, the Hall conductivity $\sigma_{y x}^{\text {tot }}$ is

$$
\sigma_{y x}^{\mathrm{tot}}=\sigma_{y x}^{\mathrm{NM}}+\sigma_{y x}^{\mathrm{SM}} .
$$

Since $\Delta \rho_{x x}^{\mathrm{NM}}(H)$ displays a nonsaturating $H^{2}$ MR for all finite $x$ [18], charge compensation must be imposed on the NM component across the entire series. According to Eq. (C2), it follows that $\rho_{y x}^{\mathrm{NM}}(H)$ is strictly $H$ linear. To estimate the form of $\rho_{y x}^{\mathrm{SM}}(H)$, we first calculate $\sigma_{y x}^{\text {tot }}(H)$ from the experimentally determined $\rho_{x x}^{\text {tot }}(H)$ [18] and $\rho_{y x}^{\text {tot }}(H)$ using the standard expression:

$$
\sigma_{y x}(H)=\frac{-\rho_{y x}(H)}{\left[\rho_{x x}(H)\right]^{2}+\left[\rho_{y x}(H)\right]^{2}} .
$$


(a)

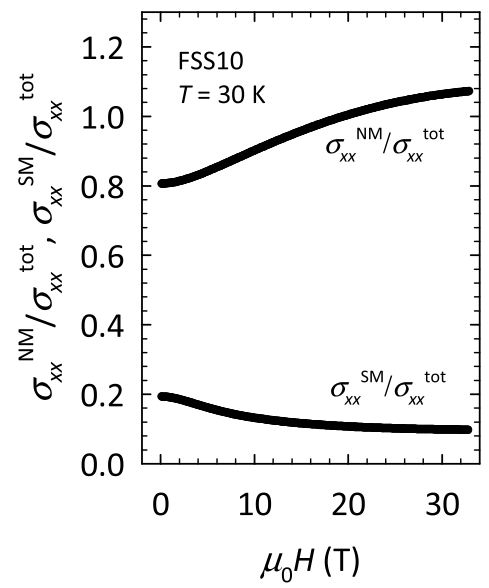

(b)

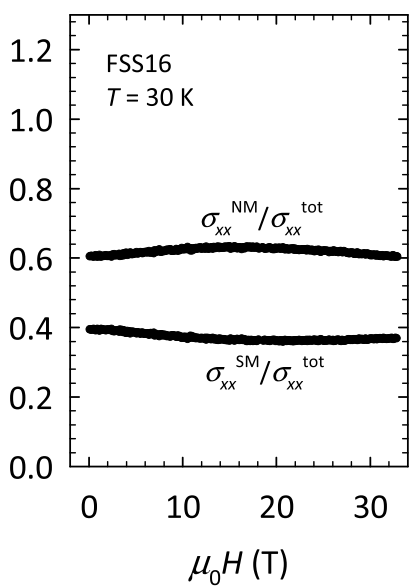

(c)

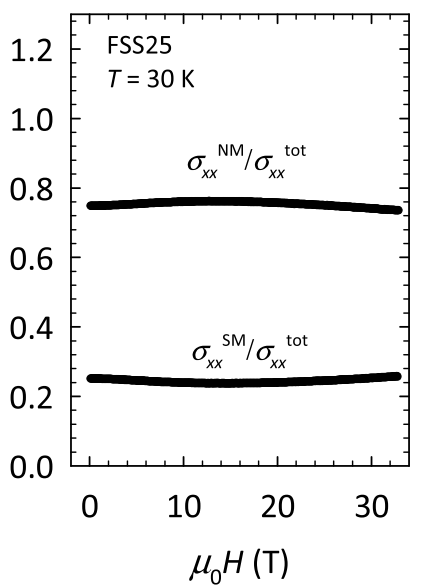

FIG. 14. Test of the validity of approximation (D8). $\sigma_{x x}^{\mathrm{NM}} / \sigma_{x x}^{\text {tot }}$ and $\sigma_{x x}^{\mathrm{SM}} / \sigma_{x x}^{\text {tot }}$ as a function of $H$ at $30 \mathrm{~K}$ for (a) FSS10, (b) FSS16, and (c) FSS25.

As explained in the main text, we assume as our starting point that $\sigma_{y x}^{\mathrm{SM}}(H)=0$ and attempt to fit $\sigma_{y x}^{\text {tot }}(H)$ with only a single NM component $\sigma_{y x}^{\mathrm{NM}}(H)$ the form of which is obtained by assuming a linear Hall resistivity $\rho_{y x}^{\mathrm{NM}}(H)=a \mu_{0} H$ and a quadratic longitudinal resistivity $\rho_{x x}^{\mathrm{NM}}(H)=b \rho_{x x}^{\text {tot }}(0)+$ $b^{2} \beta_{\mathrm{NM}}\left(\mu_{0} H\right)^{2}$ and inserting these into Eq. (E2), resulting in Eq. (1) in the main text. Here $b=\rho_{x x}^{\mathrm{NM}}(0) / \rho_{x x}^{\mathrm{tot}}(0)$ is a free fitting parameter and appears as a direct consequence of Eq. (D9). Namely, $\beta_{\mathrm{NM}}$, taken from our previous MR measurements [18], denotes the as-measured quadratic MR term and therefore, to extract the real quadratic contribution coming from the NM component, $\beta_{\mathrm{NM}}$ has to be rescaled by the weighting factor $\left[\sigma_{x x}^{\mathrm{NM}}(0) / \sigma_{x x}^{\mathrm{tot}}(0)\right]^{2}$ as imposed by Eq. (D9). Then, by examining the residual [i.e., $\sigma_{y x}^{\text {tot }}(H)-\sigma_{y x}^{\mathrm{NM}}(H)$ ], we obtain an estimate of $\sigma_{y x}^{\mathrm{SM}}(H)$. In the following sections, we describe the details of this fitting procedure and show how the resulting fit parameters also enabled us to obtain estimates of the zero-field resistivities of the two components as well as the Hall coefficient associated with the NM component.

\section{Fitting the total Hall conductivity}

There are three obvious ways with which to fit the total Hall conductivity assuming the presence of a single NM component based on Eq. (1) in the main text. The first is to fit the whole data set from 0 to $33 \mathrm{~T}$. The top panels in Fig. 15 show illustrative fits using this routine for (a) FSS13, (b) FSS16, and (c) FSS25 at $T=15 \mathrm{~K}$. While the fits are reasonable, the residuals, as one might expect, oscillate around zero and apart from the FSS13 data, display what appears to be a nonphysical form.

The alternative procedures involve restricting the fitting range to either low or high fields. The results of the low-fieldconstrained fitting procedure are shown in Figs. 15(d)-15(f) for the same data sets. In this case, the low-field feature in FSS13 forces its residual to contain the bulk of the $\sigma_{y x}(H)$ curve, in marked contrast to what is observed in the other two samples. Again, we see no physical reason for such a pronounced switch in behavior.
Restricting our fits to high fields results in the residuals shown in the main text. Other than the change in sign for $x>$ 0.13 , all residuals are found to generate $\rho_{y x}^{\mathrm{SM}}(H)$ curves with an identical form, namely, a $H$-linear growth at low fields, a peak at intermediate fields, and an exponential tail-off at high fields, similar to that obtained in a more direct manner in $\mathrm{BaFe}_{2}\left(\mathrm{As}_{1-x} \mathrm{P}_{x}\right)_{2}$ in vicinity of its AFM QCP [21]. Moreover, as shown in Fig. 6 in the main text, the evolution of $\rho_{y x}^{\mathrm{SM}}(H)$ across the nematic QCP is found to mimic that found for $\rho_{x x}^{\mathrm{SM}}(H)$ [18], suggesting that this procedure, if nothing else, generates good consistency between the measured Hall and MR responses.

It is worth noting that the high-field fitting procedure itself is to some degree arbitrary regarding the size of the highfield range over which the fitting was performed. However, as shown in Fig. 16 for FSS13 at $15 \mathrm{~K}$, the same data set shown in Fig. 3(a) in the main text, the fitting procedure performed over three different field ranges $(25 \mathrm{~T}-33 \mathrm{~T}, 18 \mathrm{~T}-33 \mathrm{~T}$, and $10 \mathrm{~T}-33 \mathrm{~T}$ ) has little influence on the residuals, which in all three cases have a pronounced maximum at $\approx 3 \mathrm{~T}$ with the value $\approx 0.8(\mathrm{~m} \Omega \mathrm{cm})^{-1}$ followed by a rapid decrease and a zero value at high fields. [An additional small feature in the middle field region, most pronounced for the shortest fitting range in Fig. 16(c), is an artefact of the fit.] Making the fitting range any bigger would again cause oscillations in the residuals, while making it smaller would introduce unnecessarily large error bars in the fitting parameters. Similar considerations also apply to the fitting of all the other samples.

\section{Details of the high-field fitting procedure}

Table I shows the fitting parameters obtained by fitting the calculated $\sigma_{y x}(H)$ data plotted in Fig. 3 to Eq. (1) for all concentrations except $x=0$. The fits themselves have two fixed parameters, $\rho_{x x}^{\text {tot }}(0)$-the measured zero-field resistivityand $\beta_{\mathrm{NM}}$-the coefficient of the (as-measured) $H^{2}$ MR term [18]-and two free parameters $a$ and $b$. Parameter $a$ is equivalent to $R_{\mathrm{H}}$ of the NM component while $b$ is equivalent to the ratio $\rho_{x x}^{\mathrm{NM}}(0) / \rho_{x x}^{\text {tot }}(0)$.

Since $a$ is always negative, $R_{\mathrm{H}}$ must be positive and thus $\mu_{h}>\mu_{e}$ for all $x$ and $T$. In most cases, $b>1$, as it should be. 
In some of the fits, particularly for FSS10, $b<1$, which is unphysical. This is a consequence of the approximations taken in obtaining Eq. (1), the most important being the approximation in Eq. (D8) that ignores the field dependence of the weighting factor $\sigma_{x x}^{\mathrm{NM}}(H) / \sigma_{x x}^{\text {tot }}(H)$ (which is directly related to the parameter $b$ ). Looking at Fig. 14(a), we can see that for FSS10 (and FSS13), $\sigma_{x x}^{\mathrm{NM}}(H) / \sigma_{x x}^{\text {tot }}(H)$ does change in magnetic field, exceeding unity at the highest fields. The case $b \leqslant 1$ prevents the decomposition of the zero-field resistivity into the NM and SM components but gives us the more flexibility in finding the unknown Hall response of the SM component. Forcing $b>1$ would make it impossible to fit the measured $\sigma_{y x}$ even in the high-field range which would cause the more complex behavior in residuals of $\sigma_{y x}$.

In cases where $b>1$, we are able to decompose $\rho_{x x}^{\text {tot }}(0)$ into NM and SM components, the results of which are listed in
Table II. As indicated, these estimates of $\rho_{x x}^{\mathrm{NM}}(0)$ and $\rho_{x x}^{\mathrm{SM}}(0)$ compare favorably with the ones extracted previously from the transverse MR [18] (listed in the fourth and fifth columns of Table II), despite the very different (and independent) way in which each ratio has been obtained.

\section{APPENDIX F: FITTING THE STRANGE METAL HALL RESISTIVITY}

To obtain $\rho_{y x}^{\mathrm{SM}}(H)$, we must first convert $\sigma_{y x}^{\mathrm{SM}}(H)$ :

$$
\rho_{y x}^{\mathrm{SM}}(H)=\frac{-\sigma_{y x}^{\mathrm{SM}}(H)}{\left[\sigma_{x x}^{\mathrm{SM}}(H)\right]^{2}+\left[\sigma_{y x}^{\mathrm{SM}}(H)\right]^{2}}
$$

(a)

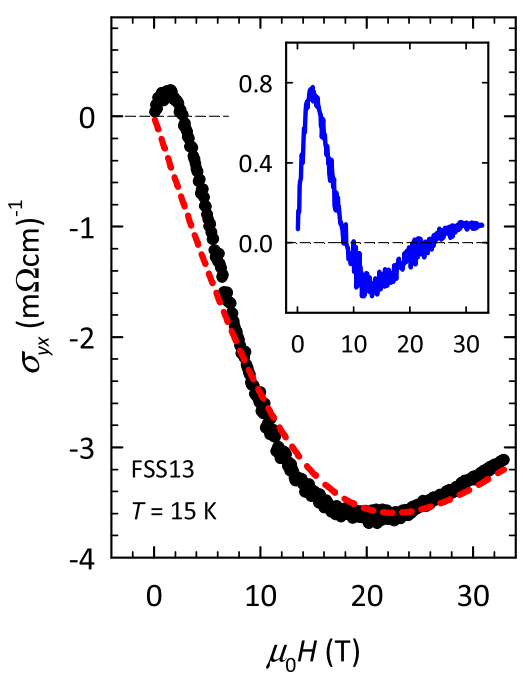

(d)

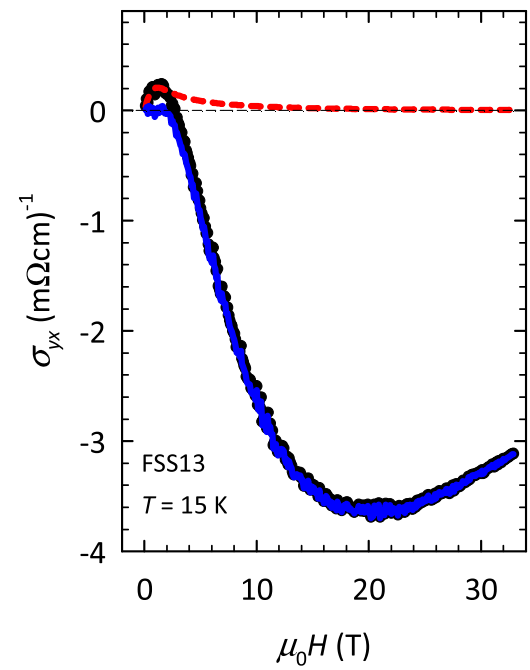

(b)

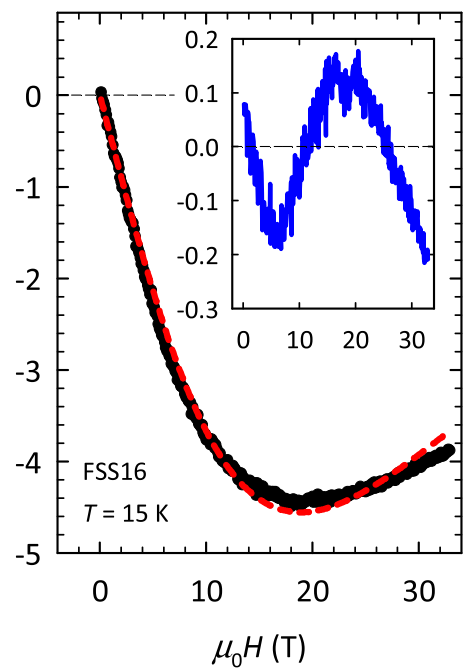

(e)

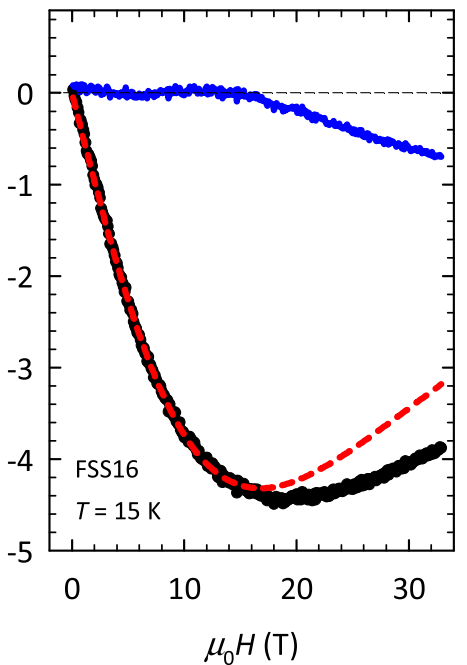

(c)

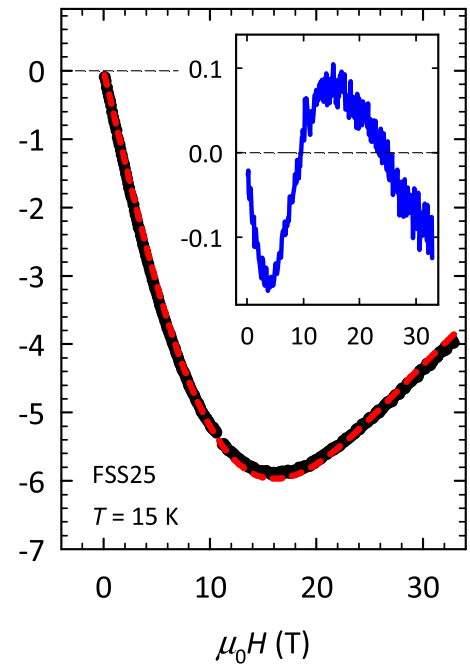

(f)

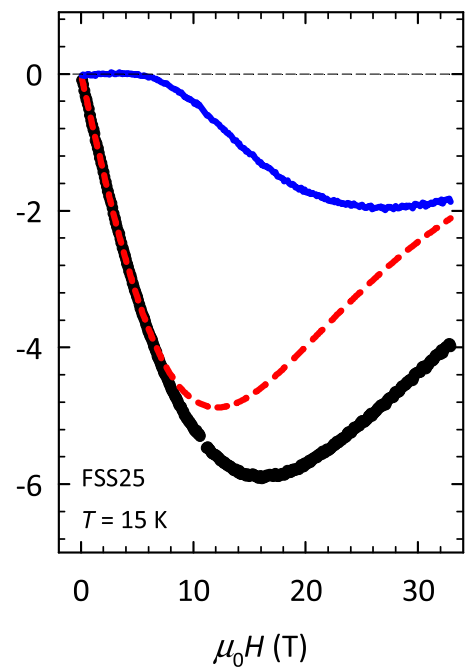

FIG. 15. (a)-(c) Fitting of $\sigma_{y x}(H)$ in $\mathrm{FeSe}_{1-x} \mathrm{~S}_{x}$ to the full field range using Eq. (1). Black dots are $\sigma_{y x}(H)$ data obtained at $T=15 \mathrm{~K}$ for (a) FSS13, (b) FSS16, and (c) FSS25. Red dashed lines represent the fits to Eq. (1) over the whole field range. The insets shows the corresponding residuals. (d)-(f) Fitting of the same $\sigma_{y x}(H)$ data for (d) FSS13, (e) FSS16, and (f) FSS25 to just the low-field regime (0-1.5 T, 0-10 T, and 0-5 T for FSS13, FSS16, and FSS25, respectively). Again, black dots are $\sigma_{y x}(H)$ data and red dashed lines the fits to Eq. (1) at low fields. Blue lines represent the corresponding fitting residuals. 
(a)

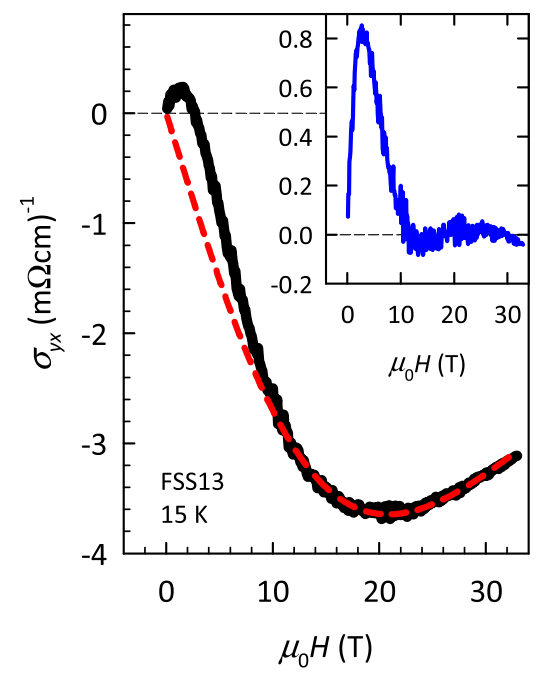

(b)

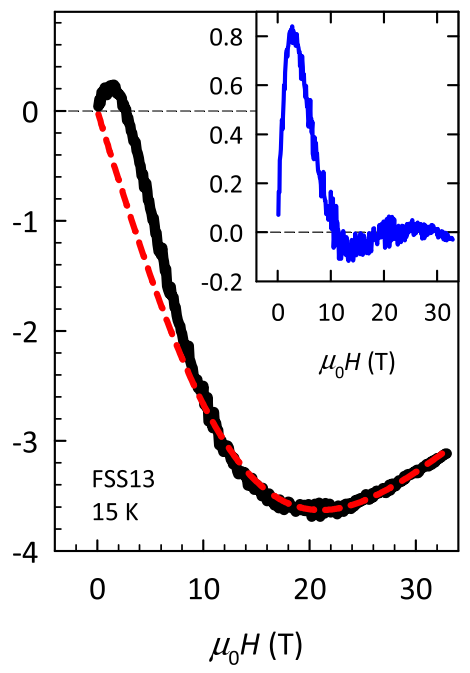

(c)

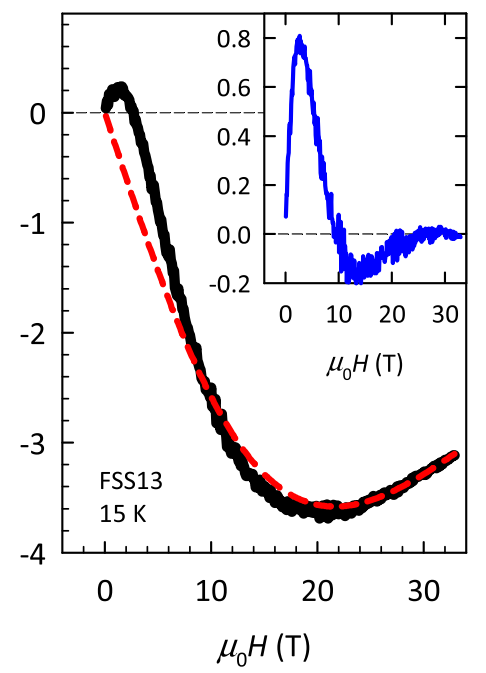

FIG. 16. High-field fitting of $\sigma_{y x}(H)$ for $\mathrm{FeSe}_{1-x} \mathrm{~S}_{x}, x=0.13$, using Eq. (1) for three different high-field ranges: (a) 10-33 T, (b) 18-33 T, and (c) 25-33 T. Black dots represent measured values and red dashed lines represent the fits to Eq. (1). The insets shows the corresponding residuals.

where $\sigma_{y x}^{\mathrm{SM}}(H)$ is given by the residuals, as shown, e.g. in Fig. 3, and:

$$
\begin{aligned}
\sigma_{x x}^{\mathrm{SM}}(H) & \approx \frac{1}{\rho_{x x}^{\mathrm{SM}}(H)} \\
& =\frac{1}{\rho_{x x}^{\mathrm{SM}}(0)+\left[\frac{\rho_{x \mathrm{xM}}^{\mathrm{SM}}(0)}{\rho_{x x}(0)}\right]^{2} \Delta \rho_{\mathrm{SM}}(H)}
\end{aligned}
$$

Here $\rho_{x x}^{\mathrm{SM}}(0)$ is the zero-field resistivity of the SM component and $\Delta \rho_{\mathrm{SM}}(H)=\Delta \rho_{\mathrm{tot}}(H)-\beta_{\mathrm{NM}}\left(\mu_{0} H\right)^{2}[18]$ is the SM contribution to the total measured MR. [The validity of the approximation in Eq. (F2) will be discussed later.] The resultant $\rho_{y x}^{\mathrm{SM}}(H)$ at representative temperatures are shown in Fig. 4 of the main text for all $x>0$. As highlighted by the solid black lines in Fig. $4, \rho_{y x}^{\mathrm{SM}}(H)$ is well described by the expression

$$
\rho_{y x}^{\mathrm{SM}}(H)=c \times \mu_{0} H \times \exp \left[-d \times\left(\mu_{0} H\right)^{2}\right],
$$

where $c$ and $d$ are free fitting parameters. All parameters relevant to this fitting procedure are listed in Tables I-III. The $R$-squared value for the majority of fits is found to be close to 1, indicating that Eq. (F3) is a reasonable approximation. However, in some cases the $R$-squared value turned out to be significantly smaller than 1, e.g., for FSS10 at $15 \mathrm{~K}$. This perhaps demonstrates that the validity of the parallel conductivity model deteriorates with decreasing $x$, as we showed in Appendix D. In addition, the quality of the fits deteriorates close to $T_{c}$ as a consequence of SC fluctuations which are visible in the zero-field resistivity [32], the longitudinal MR [18], and the Hall resistivity. For FSS10 and FSS13, where $T_{c} \approx 10 \mathrm{~K}$, it is not possible to obtain a reasonable fit over the entire magnetic field range for $T<15 \mathrm{~K}$ using Eq. (F3).

Let us here briefly discuss the validity of approximation in Eq. (F2) which enabled the determination of $\rho_{y x}^{\mathrm{SM}}(H)$ from the
Hall and longitudinal conductivity $\sigma_{y x}^{\mathrm{SM}}(H)$ and $\sigma_{x x}^{\mathrm{SM}}(H)$ data using Eq. (F1). Strictly speaking, $\sigma_{x x}^{\mathrm{SM}}(H)$ is given by

$$
\sigma_{x x}^{\mathrm{SM}}(H)=\frac{\rho_{x x}^{\mathrm{SM}}(H)}{\left[\rho_{x x}^{\mathrm{SM}}(H)\right]^{2}+\left[\rho_{y x}^{\mathrm{SM}}(H)\right]^{2}} .
$$

Note that $\rho_{y x}^{\mathrm{SM}}(H)$ also appears in Eq. (F4), making an explicit determination of $\rho_{y x}^{\mathrm{SM}}(H)$ impossible. However, if we make the assumption

$$
\frac{\left[\rho_{y x}^{\mathrm{SM}}(H)\right]^{2}}{\left[\rho_{x x}^{\mathrm{SM}}(H)\right]^{2}} \ll 1,
$$

Eq. $(\mathrm{F} 4)$ reduces to

$$
\sigma_{x x}^{\mathrm{SM}}(H) \approx \frac{1}{\rho_{x x}^{\mathrm{SM}}(H)}
$$

i.e., Eq. (F2). Looking at Fig. 4 of the main text, we can see that the maximum value of $\rho_{y x}^{\mathrm{SM}}(H)$ for FSS10 is around $70 \mu \Omega \mathrm{cm}$ at $30 \mathrm{~K}$, while the corresponding $\rho_{x x}^{\mathrm{SM}}(0) \sim$ $350 \mu \Omega \mathrm{cm}$ (see Table II) and increases with field. Hence, $\left[\rho_{y x}^{\mathrm{SM}}(H) / \rho_{x x}^{\mathrm{SM}}(0)\right]^{2} \leqslant 0.04$. Similarly, $\left[\rho_{y x}^{\mathrm{SM}}(H) / \rho_{x x}^{\mathrm{SM}}(0)\right]^{2} \sim$ 0.002-0.09 for all other concentrations, thus validating our initial assumption in Eq. (F5) and the use of the approximation (F2). In the present analysis, we used $\rho_{x x}^{\mathrm{SM}}(0)$ extracted from fitting the Hall conductivity data (third column in Table II). Only in cases where $\rho_{x x}^{\mathrm{SM}}(0)$ could not be extracted did we use the value obtained from the previous MR study [18] (fifth column in Table II).

\section{APPENDIX G: CARRIER DENSITIES AND MOBILITIES}

Having decomposed the zero-field resistivity, the longitudinal MR, and the Hall response into two contributions, we finally consider the NM component. As mentioned above, $\Delta \rho_{x x}^{\mathrm{NM}}(H)$ shows a nonsaturating $H^{2}$ dependence, which 
TABLE I. High-field fitting of the calculated $\sigma_{y x}(H)$ data based on Eq. (1). Input parameters $\rho_{x x}^{\text {tot }}(0)$ and $\beta_{\mathrm{NM}}$ are taken from Ref. [18]. Parameter $a$ is equivalent to $(-) R_{\mathrm{H}}$ of the NM component while $b$ $=\rho_{x x}^{\mathrm{NM}}(0) / \rho_{x x}^{\mathrm{tot}}(0)$.

\begin{tabular}{|c|c|c|c|c|}
\hline$T(\mathrm{~K})$ & $\begin{array}{c}\rho_{x x}^{\mathrm{tot}}(0) \\
(\mu \Omega \mathrm{cm}) \\
{[18]}\end{array}$ & $\begin{array}{c}\beta_{\mathrm{NM}} \\
\left(\mu \Omega \mathrm{cm} / \mathrm{T}^{2}\right) \\
{[18]}\end{array}$ & $\begin{array}{c}a=-R_{\mathrm{H}} \\
(\mu \Omega \mathrm{cm} / \mathrm{T})\end{array}$ & $b=\rho_{x x}^{\mathrm{NM}}(0) / \rho_{x x}^{\mathrm{tot}}(0)$ \\
\hline \multicolumn{5}{|l|}{ FSS10 } \\
\hline 30 & 61.3 & 0.022 & -0.10 & 0.76 \\
\hline 25 & 52.0 & 0.029 & -0.10 & 0.78 \\
\hline 15 & 33.3 & 0.047 & -0.15 & 0.87 \\
\hline 10 & 23.7 & 0.056 & -0.22 & 0.95 \\
\hline 6 & 16.0 & 0.063 & -0.15 & 0.77 \\
\hline 4.2 & 12.6 & 0.064 & -0.26 & 0.83 \\
\hline 1.3 & 6.7 & 0.038 & -0.60 & 1.28 \\
\hline \multicolumn{5}{|l|}{ FSS13 } \\
\hline 30 & 50.3 & 0.013 & -0.24 & 1.01 \\
\hline 25 & 44.1 & 0.014 & -0.24 & 1.02 \\
\hline 15 & 30.4 & 0.018 & -0.46 & 1.25 \\
\hline 12.5 & 26.7 & 0.018 & -0.54 & 1.29 \\
\hline 10 & 22.7 & 0.018 & -0.92 & 1.52 \\
\hline 8 & 19.7 & 0.021 & -0.60 & 1.23 \\
\hline 6 & 16.7 & 0.023 & -0.42 & 1.04 \\
\hline 4.2 & 14.5 & 0.025 & -0.35 & 0.92 \\
\hline 2.5 & 12.5 & 0.022 & -0.49 & 1.03 \\
\hline \multicolumn{5}{|l|}{ FSS16 } \\
\hline 30 & 42.9 & 0.010 & -1.23 & 1.65 \\
\hline 25 & 40.0 & 0.010 & -1.27 & 1.74 \\
\hline 15 & 30.4 & 0.010 & -0.96 & 1.71 \\
\hline 10 & 23.7 & 0.010 & -0.78 & 1.67 \\
\hline 6 & 19.9 & 0.010 & -0.91 & 1.75 \\
\hline 4.2 & 17.7 & 0.010 & -1.16 & 2.00 \\
\hline 1.3 & 14.3 & 0.008 & -2.28 & 2.70 \\
\hline \multicolumn{5}{|l|}{ FSS20 } \\
\hline 30 & 33.1 & 0.010 & -0.62 & 1.48 \\
\hline 25 & 29.3 & 0.012 & -0.66 & 1.49 \\
\hline 15 & 21.5 & 0.015 & -0.62 & 1.48 \\
\hline 10 & 16.9 & 0.017 & -0.56 & 1.43 \\
\hline 8 & 14.8 & 0.018 & -0.48 & 1.37 \\
\hline 6 & 13.0 & 0.019 & -0.38 & 1.29 \\
\hline 4.2 & 11.5 & 0.020 & -0.31 & 1.23 \\
\hline 2.5 & 10.3 & 0.023 & -0.18 & 1.06 \\
\hline \multicolumn{5}{|l|}{ FSS25 } \\
\hline 30 & 31.3 & 0.010 & -0.35 & 1.34 \\
\hline 25 & 26.6 & 0.012 & -0.33 & 1.29 \\
\hline 15 & 17.7 & 0.017 & -0.30 & 1.23 \\
\hline 10 & 13.6 & 0.019 & -0.30 & 1.24 \\
\hline 6 & 12.2 & 0.020 & -0.46 & 1.37 \\
\hline 4.2 & 11.6 & 0.020 & -0.55 & 1.43 \\
\hline 1.3 & 11.3 & 0.019 & -1.73 & 1.96 \\
\hline 0.3 & 11.2 & 0.019 & -1.45 & 1.89 \\
\hline
\end{tabular}

within the two-band model imposes the condition of perfect compensation between electrons and holes, i.e., $n_{e}=n_{h}$ and a strictly $H$-linear dependence of $\rho_{y x}^{\mathrm{NM}}(H)$. For such a compensated two-band system, it is trivial to extract the carrier densities and mobilities using Eqs. (C1) and (C2) which
TABLE II. Decomposition of $\rho_{x x}^{\text {tot }}(0)$ into NM and SM components. Columns 2 and 3 show the decomposition using parameter $b$ from Table I. Where $b \leqslant 1$, no physical value is possible. Columns 4 and 5 show corresponding values obtained from our previous MR study [18].

\begin{tabular}{|c|c|c|c|c|}
\hline$T(\mathrm{~K})$ & $\begin{array}{l}\rho_{x x}^{\mathrm{NM}}(0) \\
(\mu \Omega \mathrm{cm})\end{array}$ & $\begin{array}{l}\rho_{x x}^{\mathrm{SM}}(0) \\
(\mu \Omega \mathrm{cm})\end{array}$ & $\begin{array}{c}\rho_{x x}^{\mathrm{NM}}(0) \\
(\mu \Omega \mathrm{cm})[18]\end{array}$ & $\begin{array}{c}\rho_{x x}^{\mathrm{SM}}(0) \\
(\mu \Omega \mathrm{cm})[18]\end{array}$ \\
\hline \multicolumn{5}{|l|}{ FSS10 } \\
\hline 30 & - & - & 74.5 & 346.0 \\
\hline 25 & - & - & 64.6 & 266.6 \\
\hline 15 & - & - & 42.4 & 155.2 \\
\hline 10 & - & - & 30.2 & 110.1 \\
\hline 6 & - & - & 19.9 & 81.6 \\
\hline 4.2 & - & - & 15.3 & 71.4 \\
\hline 1.3 & 8.6 & 30.6 & 9.2 & 24.7 \\
\hline \multicolumn{5}{|l|}{ FSS13 } \\
\hline 30 & - & - & 63.1 & 248.0 \\
\hline 25 & - & - & 55.9 & 208.9 \\
\hline 15 & 38.0 & 152.0 & 38.7 & 141.7 \\
\hline 12.5 & 34.4 & 118.8 & 34.0 & 124.4 \\
\hline 10 & 34.5 & 66.4 & 28.7 & 108.6 \\
\hline 8 & 24.2 & 105.4 & 24.4 & 102.3 \\
\hline 6 & - & - & 20.4 & 92.1 \\
\hline 4.2 & - & - & 16.9 & 102.1 \\
\hline 2.5 & - & - & 14.9 & 77.6 \\
\hline \multicolumn{5}{|l|}{ FSS16 } \\
\hline 30 & 70.9 & 108.9 & 58.2 & 163.2 \\
\hline 25 & 69.6 & 94.1 & 57.5 & 131.4 \\
\hline 15 & 52.0 & 73.2 & 47.5 & 84.4 \\
\hline 10 & 39.6 & 55.0 & 39.6 & 55.0 \\
\hline 6 & 34.8 & 46.4 & 33.4 & 49.2 \\
\hline 4.2 & 35.4 & 35.4 & 33.6 & 37.4 \\
\hline 1.3 & 38.6 & 22.7 & 30.3 & 27.1 \\
\hline \multicolumn{5}{|l|}{ FSS20 } \\
\hline 30 & 49.0 & 102.1 & 38.8 & 225.3 \\
\hline 25 & 43.6 & 89.1 & 34.5 & 194.4 \\
\hline 15 & 31.8 & 66.3 & 26.0 & 124.2 \\
\hline 10 & 24.2 & 56.2 & 20.1 & 106.2 \\
\hline 8 & 20.3 & 54.8 & 17.8 & 87.8 \\
\hline 6 & 16.8 & 57.8 & 15.9 & 71.3 \\
\hline 4.2 & 14.1 & 61.5 & 14.2 & 60.5 \\
\hline 2.5 & - & - & 13.3 & 45.7 \\
\hline \multicolumn{5}{|l|}{ FSS25 } \\
\hline 30 & 41.9 & 123.4 & 39.3 & 153.8 \\
\hline 25 & 34.3 & 118.3 & 33.3 & 132.2 \\
\hline 15 & 21.8 & 94.7 & 21.4 & 102.4 \\
\hline 10 & 16.9 & 70.3 & 17.2 & 65.0 \\
\hline 6 & 16.7 & 45.2 & 15.0 & 65.4 \\
\hline 4.2 & 16.6 & 38.6 & 14.4 & 59.7 \\
\hline 1.3 & 22.1 & 23.1 & 14.5 & 51.2 \\
\hline 0.3 & 21.2 & 23.8 & 15.6 & 39.7 \\
\hline
\end{tabular}

reduce to

$$
\begin{aligned}
\sigma & =n e\left(\mu_{e}+\mu_{h}\right), \\
R_{\mathrm{H}} & =\frac{1}{n e} \frac{\mu_{h}-\mu_{e}}{\mu_{h}+\mu_{e}}, \\
\frac{\Delta \rho}{\rho_{0}} & =\mu_{h} \mu_{e}\left(\mu_{0} H\right)^{2},
\end{aligned}
$$


TABLE III. Fitting of $\rho_{y x}^{\mathrm{SM}}$ based on Eq. (F3). Columns 2 and 3 show the fitting parameters $c$ and $d$ and the last column shows goodness of fit with the standard $R$-squared $\left(R_{\text {sqr }}\right)$ value.

\begin{tabular}{|c|c|c|c|}
\hline$T(\mathrm{~K})$ & $c(\mu \Omega \mathrm{cm} / \mathrm{T})$ & $d\left(\mathrm{~T}^{-2}\right)$ & $R_{\mathrm{sqr}}$ \\
\hline \multicolumn{4}{|l|}{ FSS10 } \\
\hline 30 & -12.4 & 0.0059 & 0.9701 \\
\hline 25 & -12.8 & 0.0063 & 0.9666 \\
\hline 15 & -10.2 & 0.032 & 0.5557 \\
\hline \multicolumn{4}{|l|}{ FSS13 } \\
\hline 30 & -12.3 & 0.0059 & 0.9693 \\
\hline 25 & -13.3 & 0.0069 & 0.9556 \\
\hline 15 & -13.7 & 0.025 & 0.7160 \\
\hline \multicolumn{4}{|l|}{ FSS16 } \\
\hline 30 & 0.58 & 0.0068 & 0.8095 \\
\hline 25 & 0.63 & 0.0074 & 0.8373 \\
\hline 15 & 1.1 & 0.0048 & 0.9140 \\
\hline 10 & 2.0 & 0.0049 & 0.9039 \\
\hline 6 & 3.1 & 0.0059 & 0.9355 \\
\hline \multicolumn{4}{|l|}{ FSS20 } \\
\hline 30 & 0.38 & 0.0082 & 0.8368 \\
\hline 25 & 0.48 & 0.0092 & 0.8793 \\
\hline 15 & 0.80 & 0.010 & 0.9231 \\
\hline 10 & 0.97 & 0.014 & 0.8859 \\
\hline 8 & 1.2 & 0.015 & 0.8287 \\
\hline \multicolumn{4}{|l|}{ FSS25 } \\
\hline 30 & 0.42 & 0.0089 & 0.8620 \\
\hline 25 & 0.70 & 0.011 & 0.8863 \\
\hline 15 & 0.86 & 0.010 & 0.8851 \\
\hline 10 & 0.49 & 0.015 & 0.7658 \\
\hline
\end{tabular}

where $\sigma=1 / \rho_{x x}^{\mathrm{NM}}(0), n=n_{e}=n_{h}, R_{\mathrm{H}}=\rho_{y x}^{\mathrm{NM}} /\left(\mu_{0} H\right)$, and

$$
\begin{aligned}
\frac{\Delta \rho}{\rho_{0}} & =\frac{\rho_{x x}^{\mathrm{NM}}(H)-\rho_{x x}^{\mathrm{NM}}(0)}{\rho_{x x}^{\mathrm{NM}}(0)} \\
& =\frac{\left[\frac{\rho_{x x}^{\mathrm{NM}}(0)}{\rho_{x x}^{\mathrm{tot}}(0)}\right]^{2} \beta_{\mathrm{NM}}\left(\mu_{0} H\right)^{2}}{\rho_{x x}^{\mathrm{NM}}(0)} .
\end{aligned}
$$

The extracted values are given in Table IV. The carrier mobilities are in the range $200-1000 \mathrm{~cm}^{2} / \mathrm{Vs}$ while the carrier densities range from $2-4 \times 10^{20} \mathrm{~cm}^{-3}$ or $0.016-0.032$ carriers per Fe atom for all $T$ and $x$ studied. We find both mobilities increase with decreasing temperature, reflecting the overall metallic behavior, while $\mu_{h}>\mu_{e}$ at all $T$. The carrier densities do not exhibit any noticeable $T$ dependence and, as shown in Fig. 5 of the main text, their average value for each $x$ is approximately half those extracted from a recent QO study [23].

Finally, we compare the conclusions drawn from our present analysis with those of the mobility spectrum analysis performed on pure FeSe by Huynh et al. [53]. The latter led to the appearance of three mobility peaks in pure FeSe; two ascribed to the large, almost perfectly compensated electron and hole pockets and one to a tiny electron pocket having a much larger carrier mobility. This small pocket was believed to originate either from a Dirac cone or a large anisotropy of the FS. Our analysis confirms that there is a contribution com-
TABLE IV. Calculated carrier densities and mobilities in $\mathrm{FeSe}_{1-x} \mathrm{~S}_{x}$. The values were determined by applying the compensated two-band model [Eqs. (G1)-(G3)] on zero-field resistivity, MR, and Hall coefficient extracted from the NM component as described in the main text. The last column gives the carrier density per Fe atom calculated by taking into account the unit cell volume $V_{\text {cell }}=78.2 \AA^{3}$ [50] for the tetragonal structure and the fact that there is one formula

\begin{tabular}{|c|c|c|c|c|}
\hline$T(\mathrm{~K})$ & $\mu_{h}\left(\mathrm{~cm}^{2} / \mathrm{Vs}\right)$ & $\mu_{e}\left(\mathrm{~cm}^{2} / \mathrm{Vs}\right)$ & $n\left(10^{20} \mathrm{~cm}^{-3}\right)$ & $n($ per Fe $)$ \\
\hline \multicolumn{5}{|l|}{ FSS10 } \\
\hline 30 & 216.7 & 204.4 & 2 & 0.0156 \\
\hline 25 & 269.7 & 254.3 & 1.8 & 0.0140 \\
\hline 15 & 442.4 & 406.9 & 1.7 & 0.0132 \\
\hline 10 & 585.4 & 512.4 & 1.9 & 0.0148 \\
\hline 6 & 739.0 & 663.0 & 2.3 & 0.0180 \\
\hline 4.2 & 881.6 & 714.6 & 2.5 & 0.0196 \\
\hline \multicolumn{5}{|l|}{ FSS13 } \\
\hline 30 & 197.8 & 159.8 & 2.8 & 0.0220 \\
\hline 25 & 222.6 & 179.7 & 2.8 & 0.0220 \\
\hline 15 & 336.8 & 216.8 & 3 & 0.0240 \\
\hline 12.5 & 384.7 & 228.7 & 3 & 0.0240 \\
\hline 10 & 512.7 & 245.7 & 2.4 & 0.0188 \\
\hline 8 & 505.3 & 257.3 & 3.4 & 0.0260 \\
\hline 6 & 525.6 & 319.6 & 3.6 & 0.0280 \\
\hline \multicolumn{5}{|l|}{ FSS16 } \\
\hline 30 & 301.2 & 128.2 & 2.1 & 0.0164 \\
\hline 25 & 320.1 & 137.1 & 2 & 0.0156 \\
\hline 15 & 351.5 & 166.5 & 2.3 & 0.0181 \\
\hline 10 & 380.8 & 183.8 & 2.8 & 0.0220 \\
\hline 6 & 454.5 & 192.5 & 2.8 & 0.0220 \\
\hline 4.2 & 537.3 & 210.3 & 2.7 & 0.0212 \\
\hline \multicolumn{5}{|l|}{ FSS20 } \\
\hline 30 & 288.8 & 162.8 & 2.8 & 0.0220 \\
\hline 25 & 333.2 & 182.2 & 2.8 & 0.0220 \\
\hline 15 & 435.1 & 239.1 & 2.9 & 0.0226 \\
\hline 10 & 512.8 & 282.8 & 3.2 & 0.0250 \\
\hline 8 & 542.3 & 304.3 & 3.6 & 0.0282 \\
\hline 6 & 560.9 & 329.9 & 4.2 & 0.0328 \\
\hline 4.2 & 585.2 & 368.8 & 4.6 & 0.0360 \\
\hline 2.5 & 604.7 & 464.7 & 4.5 & 0.0352 \\
\hline \multicolumn{5}{|l|}{ FSS25 } \\
\hline 30 & 258.0 & 173.7 & 3.5 & 0.0274 \\
\hline 25 & 295.9 & 199.4 & 3.7 & 0.0290 \\
\hline 15 & 413.9 & 277.9 & 4.2 & 0.0328 \\
\hline 10 & 510.8 & 332.8 & 4.4 & 0.0344 \\
\hline 6 & 633.4 & 358.4 & 3.8 & 0.0298 \\
\hline 4.2 & 696.2 & 366.2 & 3.5 & 0.0274 \\
\hline
\end{tabular}
unit per cell.

ing from a pair of compensated electron and hole pockets with mobilities and densities very similar to the ones found in the mobility spectrum analysis [53], plus an extra contribution, the behavior of which is similar to that found in other quantum critical systems close to a QCP [14-17,19]. Since this additional contribution is both universal and exhibits a systematic evolution across $x_{c}$, we ascribe it here to the SM component, rather than to Dirac fermions or to a large anisotropy in the FS parameters, all of which are expected to disappear beyond $x_{c}$. 
[1] S. Sachdev, Quantum Phase Transitions (Cambridge University Press, Cambridge, UK, 2011).

[2] H. Löhneysen, S. Mock, A. Neubert, T. Pietrus, A. Rosch, A. Schröder, O. Stockert, and U. Tutsch, Heavy-fermion systems at the magnetic-nonmagnetic quantum phase transition, J. Magn. Magn. Mater. 177-181, 12 (1998).

[3] J. Custers, P. Gegenwart, H. Wilhelm, K. Neumaier, Y. Tokiwa, O. Trovarelli, C. Geibel, F. Steglich, C. Pépin, and P. Coleman, The break-up of heavy electrons at a quantum critical point, Nature 424, 524 (2003).

[4] J. A. N. Bruin, H. Sakai, R. S. Perry, and A. P. Mackenzie, Similarity of scattering rates in metals showing $T$-linear resistivity, Science 339, 804 (2013).

[5] N. E. Hussey, K. Takenaka, and H. Takagi, Universality of the Mott-Ioffe-Regel limit in metals, Philos. Mag. 84, 2847 (2004).

[6] J. Zaanen, Why the temperature is high, Nature 430, 512 (2004).

[7] S. A. Hartnoll, Theory of universal incoherent metallic transport, Nat. Phys. 11, 54 (2015).

[8] J. G. Analytis, H.-H. Kuo, R. D. McDonald, M. Wartenbe, P. M. C. Rourke, N. E. Hussey, and I. R. Fisher, Transport near a quantum critical point in $\mathrm{BaFe}_{2}\left(\mathrm{As}_{1-x} \mathrm{P}_{x}\right)_{2}$, Nat. Phys. 10, 194 (2014).

[9] T. R. Chien, Z. Z. Wang, and N. P. Ong, Effect of Zn Impurities on the Normal-State Hall Angle in Single-Crystal $\mathrm{YBa}_{2} \mathrm{Cu}_{3-x} \mathrm{Zn}_{x} \mathrm{O}_{7-\delta}$, Phys. Rev. Lett. 67, 2088 (1991).

[10] Y. Nakajima, H. Shishido, H. Nakai, T. Shibauchi, K. Behnia, K. Izawa, M. Hedo, Y. Uwatoko, T. Matsumoto, R. Settai, Y. Onuki, H. Kontani, and Y. Matsuda, Non-Fermi liquid behavior in the magnetotransport of $\mathrm{Ce} \mathrm{In}_{5}$ ( $M$ : Co and $\left.\mathrm{Rh}\right)$ : Striking similarity between quasi two-dimensional heavy fermions and high- $T_{C}$ cuprates, J. Phys. Soc. Jpn. 76, 024703 (2007).

[11] R. H. Liu, G. Wu, T. Wu, D. F. Fang, H. Chen, S. Y. Li, K. Liu, Y. L. Xie, X. F. Wang, R. L. Yang, L. Ding, C. He, D. L. Feng, and X. H. Chen, Anomalous Transport Properties and Phase Diagram of the FeAs-Based $\mathrm{SmFeAsO}_{1-x} \mathrm{~F}_{x}$ superconductors, Phys. Rev. Lett. 101, 087001 (2008).

[12] J. M. Harris, Y. F. Yan, P. Matl, N. P. Ong, P. W. Anderson, T. Kimura, and K. Kitazawa, Violation of Kohler's Rule in the Normal-State Magnetoresistance of $\mathrm{YBa}_{2} \mathrm{Cu}_{3} \mathrm{O}_{7-\delta}$ and $\mathrm{La}_{2-x} \mathrm{Sr}_{x} \mathrm{CuO}_{4}$, Phys. Rev. Lett. 75, 1391 (1995).

[13] S. Kasahara, T. Shibauchi, K. Hashimoto, K. Ikada, S. Tonegawa, R. Okazaki, H. Shishido, H. Ikeda, H. Takeya, K. Hirata, T. Terashima, and Y. Matsuda, Evolution from non-Fermi- to Fermi-liquid transport via isovalent doping in $\mathrm{BaFe}_{2}\left(\mathrm{As}_{1-x} \mathrm{P}_{x}\right)_{2}$ superconductors, Phys. Rev. B 81, 184519 (2010).

[14] I. M. Hayes, R. D. McDonald, N. P. Breznay, T. Helm, P. J. W. Moll, M. Wartenbe, A. Shekhter, and J. G. Analytis, Scaling between magnetic field and temperature in the high-temperature superconductor $\mathrm{BaFe}_{2}\left(\mathrm{As}_{1-x} \mathrm{P}_{x}\right)_{2}$, Nat. Phys. 12, 916 (2016).

[15] I. M. Hayes, Z. Hao, N. Maksimovic, S. K. Lewin, M. K. Chan, R. D. McDonald, B. J. Ramshaw, J. E. Moore, and J. G. Analytis, Magnetoresistance in $\mathrm{BaFe}_{2}\left(\mathrm{As}_{1-x} \mathrm{P}_{x}\right)_{2}$, Phys. Rev. Lett. 121, 197002 (2018).

[16] P. Giraldo-Gallo, J. A. Galvis, Z. Stegen, K. A. Modic, F. F. Balakirev, J. B. Betts, X. Lian, C. Moir, S. C. Riggs, J. Wu, A. T. Bollinger, X. He, I. Božović, B. J. Ramshaw, R. D. McDonald, G. S. Boebinger, and A. Shekhter, Scale-invariant magnetoresistance in a cuprate superconductor, Science 361, 479 (2018).
[17] T. Sarkar, P. R. Mandal, N. R. Poniatowski, M. K. Chan, and R. L. Greene, Correlation between scale-invariant normal-state resistivity and superconductivity in an electron-doped cuprate, Sci. Adv. 5, eaav6753 (2019).

[18] S. Licciardello, N. Maksimovic, J. Ayres, J. Buhot, M. Čulo, B. Bryant, S. Kasahara, Y. Matsuda, T. Shibauchi, V. Nagarajan, J. G. Analytis, and N. E. Hussey, Coexistence of orbital and quantum critical magnetoresistance in $\mathrm{FeSe}_{1-x} \mathrm{~S}_{x}$, Phys. Rev. Research 1, 023011 (2019).

[19] J. Ayres, M. Berben, M. Čulo, Y. Hsu, E. van Heumen, Y. Huang, J. Zaanen, T. Kondo, T. Takeuchi, J. R. Cooper, C. Putzke, S. Friedemann, A. Carrington, and N. E. Hussey, Incoherent transport across the strange metal regime of highly overdoped cuprates, arXiV:2012.01208.

[20] C. Putzke, S. Benhabib, W. Tabis, J. Ayres, Z. Wang, L. Malone, S. Licciardello, J. Lu, T. Kondo, T. Takeuchi, N. E. Hussey, J. R. Cooper, and A. Carrington, Reduced Hall carrier density in the overdoped strange metal regime of cuprate superconductors, Nat. Phys. (2021), doi:10.1038/s41567-021-01197-0.

[21] I. M. Hayes, N. Maksimovic, G. N. Lopez, M. K. Chan, B. J. Ramshaw, R. D. McDonald, and J. G. Analytis, Superconductivity and quantum criticality linked by the Hall effect in a strange metal, Nat. Phys. 17, 58 (2021).

[22] W. K. Huang, S. Hosoi, M. Čulo, S. Kasahara, Y. Sato, K. Matsuura, Y. Mizukami, M. Berben, N. E. Hussey, H. Kontani, T. Shibauchi, and Y. Matsuda, Non-Fermi liquid transport in the vicinity of the nematic quantum critical point of superconducting $\mathrm{FeSe}_{1-x} \mathrm{~S}_{x}$, Phys. Rev. Research 2, 033367 (2020).

[23] A. I. Coldea, S. F. Blake, S. Kasahara, A. A. Haghighirad, M. D. Watson, W. Knafo, E. S. Choi, A. McCollam, P. Reiss, T. Yamashita, M. Bruma, S. C. Speller, Y. Matsuda, T. Wolf, T. Shibauchi, and A. J. Schofield, Evolution of the low-temperature Fermi surface of superconducting $\mathrm{FeSe}_{1-x} \mathrm{~S}_{x}$ across a nematic phase transition, npj Quantum Mater. 4, 2 (2019).

[24] A. E. Böhmer and A. Kriesel, Nematicity, magnetism and superconductivity in FeSe, J. Phys.: Condens. Matter 30, 023001 (2018).

[25] A. I. Coldea and M. D. Watson, The key ingredients of the electronic structure of FeSe, Annu. Rev. Condens. Matter Phys. 9, 125 (2018).

[26] A. Kriesel, P. J. Hirschfeld, and B. M. Andersen, On the remarkable superconductivity of FeSe and its close cousins, Symmetry 12, 1402 (2020).

[27] T. Shibauchi, T. Hanaguri, and Y. Matsuda, Exotic superconducting states in FeSe-based materials, J. Phys. Soc. Jpn. 89, 102002 (2020).

[28] S. Hosoi, K. Matsuura, K. Ishida, H. Wang, Y. Mizukami, T. Watashige, S. Kasahara, Y. Matsuda, and T. Shibauchi, Nematic quantum critical point without magnetism in $\mathrm{FeSe}_{1-x} \mathrm{~S}_{x}$ superconductors, Proc. Natl. Acad. Sci. 113, 8139 (2016).

[29] T. Hanaguri, K. Iwaya, Y. Kohsaka, T. Machida, T. Watashige, S. Kasahara, T. Shibauchi, and Y. Matsuda, Two distinct superconducting pairing states divided by the nematic end point in $\mathrm{FeSe}_{1-x} \mathrm{~S}_{x}$, Sci. Adv. 4, eaar6419 (2018).

[30] Y. Sato, S. Kasahara, T. Taniguchi, X. Xing, Y. Kasahara, Y. Tokiwa, Y. Yamakawa, H. Kontani, T. Shibauchi, and Y. Matsuda, Abrupt change of the superconducting gap structure at the nematic critical point in $\mathrm{FeSe}_{1-x} \mathrm{~S}_{x}$, Proc. Natl. Acad. Sci. 115, 1227 (2018). 
[31] M. Bristow, P. Reiss, A. A. Haghighirad, Z. Zajicek, S. J. Singh, T. Wolf, D. Graf, W. Knafo, A. McCollam, and A. I. Coldea, Anomalous high-magnetic field electronic state of the nematic superconductors $\mathrm{FeSe}_{1-x} \mathrm{~S}_{x}$, Phys. Rev. Research 2, 013309 (2020).

[32] S. Licciardello, J. Buhot, J. Lu, J. Ayres, S. Kasahara, Y. Matsuda, T. Shibauchi, and N. E. Hussey, Electrical resistivity across a nematic quantum critical point, Nature 567, 213 (2019).

[33] T. Urata, Y. Tanabe, K.-K. Huynh, H. Oguro, K. Watanabe, and K. Tanigaki, Non-Fermi liquid behavior of electrical resistivity close to the nematic critical point in $\mathrm{Fe}_{1-x} \mathrm{Co}_{x} \mathrm{Se}$ and $\mathrm{FeSe}_{1-y} \mathrm{~S}_{y}$, arXiV:1608.01044.

[34] M. A. Tanatar, A. E. Böhmer, E. I. Timmons, M. Schütt, G. Drachuck, V. Taufour, K. Kothapalli, A. Kreyssig, S. L. Bud'ko, P. C. Canfield, R. M. Fernandes, and R. Prozorov, Origin of the Resistivity Anisotropy in the Nematic Phase of FeSe, Phys. Rev. Lett. 117, 127001 (2016).

[35] I. Paul and M. Garst, Lattice Effects on Nematic Quantum Criticality in Metals, Phys. Rev. Lett. 118, 227601 (2017).

[36] P. Reiss, D. Graf, A. A. Haghighirad, W. Knafo, L. Drigo, M. Bristow, A. J. Schofield, and A. I. Coldea, Quenched nematic criticality and two superconducting domes in an iron-based superconductor, Nat. Phys. 16, 89 (2020).

[37] K. Matsuura, Y. Mizukami, Y. Arai, Y. Sugimura, N. Maejima, A. Machida, T. Watanuki, T. Fukuda, T. Yajima, Z. Hiroi, K. Y. Yip, Y. C. Chan, Q. Niu, S. Hosoi, K. Ishida, K. Mukasa, S. Kasahara, J.-G. Cheng, S. K. Goh, Y. Matsuda, Y. Uwatoko, and T. Shibauchi, Maximizing $T_{C}$ by tuning nematicity and magnetism in $\mathrm{FeSe}_{1-x} \mathrm{~S}_{x}$ superconductors, Nat. Commun. 8, 1143 (2017).

[38] P. Wiecki, K. Rana, A. E. Böhmer, Y. Lee, S. L. Bud'ko, P. C. Canfield, and Y. Furukawa, Persistent correlation between superconductivity and antiferromagnetic fluctuations near a nematic quantum critical point in $\mathrm{FeSe}_{1-x} \mathrm{~S}_{x}$, Phys. Rev. B 98, 020507(R) (2018).

[39] See Appendix B and Figs. 8 and 9 for more detail.

[40] For more details about the determination of $T_{s}$, see Appendix A.

[41] F. Hardy, M. He, L. Wang, T. Wolf, P. Schweiss, M. Merz, M. Barth, P. Adelmann, R. Eder, A.-A. Haghighirad, and C. Meingast, Calorimetric evidence of nodal gaps in the nematic superconductor FeSe, Phys. Rev. B 99, 035157 (2019).

[42] M. Abdel-Hafiez, Y. J. Pu, J. Brisbois, R. Peng, D. L. Feng, D. A. Chareev, A. V. Silhanek, C. Krellner, A. N. Vasiliev, and X.-J. Chen, Impurity scattering effects on the superconducting properties and the tetragonal-to-orthorhombic phase transition in FeSe, Phys. Rev. B 93, 224508 (2016).

[43] D. Chareev, Y. Ovchenkov, L. Shvanskaya, A. Kovalskii, M. Abdel-Hafiez, D. J. Trainer, E. M. Lechner, M. Iavarone, O. Volkova, and A. Vasiliev, Single crystal growth, transport and scanning tunneling microscopy and spectroscopy of $\mathrm{FeSe}_{1-x} \mathrm{~S}_{x}$, CrystEngComm 20, 2449 (2018).

[44] M. D. Watson, T. Yamashita, S. Kasahara, W. Knafo, M. Nardone, J. Béard, F. Hardy, A. McCollam, A. Narayanan, S. F. Blake, T. Wolf, A. A. Haghighirad, C. Meingast, A. J. Schofield, H. v. Löhneysen, Y. Matsuda, A. I. Coldea, and T. Shibauchi, Dichotomy between the Hole and Electron Behavior in Multiband Superconductor FeSe Probed by Ultrahigh Magnetic Fields, Phys. Rev. Lett. 115, 027006 (2015).
[45] M. D. Watson, A. A. Haghighirad, L. C. Rhodes, M. Hoesch, and T. K. Kim, Electronic anisotropies revealed by detwinned angle-resolved photo-emission spectroscopy measurements of FeSe, New J. Phys. 19, 103021 (2017).

[46] M. Yi, H. Pfau, Y. Zhang, Y. He, H. Wu, T. Chen, Z. R. Ye, M. Hashimoto, R. Yu, Q. Si, D.-H. Lee, P. Dai, Z.-X. Shen, D. H. Lu, and R. J. Birgeneau, Nematic Energy Scale and the Missing Electron Pocket in FeSe, Phys. Rev. X 9, 041049 (2019).

[47] S. S. Huh, J. J. Seo, B. S. Kim, S. H. Cho, J. K. Jung, S. Kim, C. I. Kwon, J. S. Kim, Y. Y. Koh, W. S. Kyung, J. D. Denlinger, Y. H. Kim, B. N. Chae, N. D. Kim, Y. K. Kim, and C. Kim, Absence of Y-pocket in 1-Fe Brillouin zone and reversed orbital occupation imbalance in FeSe, Commun. Phys. 3, 52 (2020).

[48] L. C. Rhodes, M. D. Watson, A. A. Haghighirad, D. V. Evtushinsky, and T. K. Kim, Revealing the single electron pocket of FeSe in a single orthorhombic domain, Phys. Rev. B 101, 235128 (2020).

[49] T. Terashima, N. Kikugawa, A. Kiswandhi, E.-S. Choi, J. S. Brooks, S. Kasahara, T. Watashige, H. Ikeda, T. Shibauchi, Y. Matsuda, T. Wolf, A. E. Böhmer, F. Hardy, C. Meingast, H. v. Löhneysen, M.-T. Suzuki, R. Arita, and S. Uji, Anomalous Fermi surface in FeSe seen by Shubnikov de Haas oscillation measurements, Phys. Rev. B 90, 144517 (2014).

[50] M. D. Watson, T. K. Kim, A. A. Haghighirad, N. R. Davies, A. McCollam, A. Narayanan, S. F. Blake, Y. L. Chen, S. Ghannadzadeh, A. J. Schofield, M. Hoesch, C. Meingast, T. Wolf, and A. I. Coldea, Emergence of the nematic electronic state in FeSe, Phys. Rev. B 91, 155106 (2015).

[51] J.-Y. Lin, Y. S. Hsieh, D. A. Chareev, A. N. Vasiliev, Y. Parsons, and H. D. Yang, Coexistence of isotropic and extended s-wave order parameters in FeSe as revealed by low-temperature specific heat, Phys. Rev. B 84, 220507(R) (2011).

[52] A. Böhmer, T. Arai, F. Hardy, T. Hattori, T. Iye, T. Wolf, H. Löhneysen, K. Ishida, and C. Meingast, Origin of the Tetragonal-To-Orthorhombic Phase Transition in FeSe: A Combined Thermodynamic and NMR Study of Nematicity, Phys. Rev. Lett. 114, 027001 (2015).

[53] K. K. Huynh, Y. Tanabe, T. Urata, H. Oguro, S. Heguri, K. Watanabe, and K. Tanigaki, Electric transport of a singlecrystal iron chalcogenide FeSe superconductor: Evidence of symmetry-breakdown nematicity and additional ultrafast Dirac cone-like carriers, Phys. Rev. B 90, 144516 (2014).

[54] L. C. Rhodes, J. M. A. Böker, Müller, M. Eschrig, and I. M. Eremin, Non-local dxy nematicity and the missing electron pocket in FeSe, arXiv:2009.00507.

[55] See Eqs. (C3) through (C6) in Appendix C.

[56] J. S. Kim, A matrix formalism for the Hall effect in multicarrier semiconductor systems, J. Appl. Phys. 86, 3187 (1999).

[57] For more details about the two- and three-band model fittings, see Appendix C.

[58] S. Ishida, T. Liang, M. Nakajima, K. Kihou, C. H. Lee, A. Iyo, H. Eisaki, T. Kakeshita, T. Kida, M. Hagiwara, Y. Tomioka, T. Ito, and S. Uchida, Manifestations of multiplecarrier charge transport in the magnetostructurally ordered phase of $\mathrm{BaFe}_{2} \mathrm{As}_{2}$, Phys. Rev. B 84, 184514 (2011).

[59] K. K. Huynh, Y. Tanabe, and K. Tanigaki, Both Electron and Hole Dirac Cone States in $\mathrm{BaFeAs}_{2}$ Confirmed by Magnetoresistance, Phys. Rev. Lett. 106, 217004 (2011). 
[60] H.-H. Kuo, J.-H. Chu, S. C. Riggs, L. Yu, P. L. McMahon, K. De Greve, Y. Yamamoto, J. G. Analytis, and I. R. Fisher, Possible origin of the nonmonotonic doping dependence of the in-plane resistivity anisotropy of $\mathrm{Ba}\left(\mathrm{Fe}_{1-x} T_{x}\right)_{2} \mathrm{As}_{2}(T=\mathrm{Co}, \mathrm{Ni}$ and $\mathrm{Cu})$, Phys. Rev. B 84, 054540 (2011).

[61] A. A. Abrikosov, Quantum magnetoresistance, Phys. Rev. B 58, 2788 (1998).

[62] Y. Sun, S. Pyon, and T. Tamegai, Electron carriers with possible Dirac-cone-like dispersion in $\mathrm{FeSe}_{1-x} \mathrm{~S}_{x}(x=0$ and 0.14) single crystals triggered by structural transition, Phys. Rev. B 93, 104502 (2016).

[63] S. Y. Tan, Y. Fang, D. H. Xie, W. Feng, C. H. P. Wen, Q. Song, Q. Y. Chen, W. Zhang, Y. Zhang, L. Z. Luo, B. P. Xie, X. C. Lai, and D. L. Feng, Observation of Dirac cone band dispersions in FeSe thin films by photoemission spectroscopy, Phys. Rev. B 93, 104513 (2016).

[64] A. Kostin, P. O. Sprau, A. Kreisel, Y. X. Chong, A. E. Böhmer, P. C. Canfield, P. J. Hirschfeld, B. M. Andersen, and J. C. S. Davis, Imaging orbital-selective quasiparticles in the Hunds metal state of FeSe, Nat. Mater. 17, 869 (2018).

[65] In pure FeSe, where the mobilities are much greater, the MR is found to be composed of a quadrature term plus a second term that deviates from a $H^{2}$ dependence above around $10 \mathrm{~T}$ [18]. Nevertheless, this second term can still be fitted using standard two-band analysis.

[66] The measured data are calculated using Eq. (E2) in Appendix E.

[67] The derivation of Eq. (1) is given in Appendix E.

[68] The free fitting parameter $b$ is related to the weighting factor in Eq. (D9) in Appendix D.

[69] N. Maksimovic, I. M. Hayes, V. Nagarajan, J. G. Analytis, A. E. Koshelev, J. Singleton, Y.-B. Lee, and T. Schenkel, Magnetoresistance Scaling and the Origin of H-Linear Resistivity in $\mathrm{BaFe}_{2}\left(\mathrm{As}_{1-x} \mathrm{P}_{x}\right)_{2}$, Phys. Rev. X 10, 041062 (2020).

[70] As shown in Appendix E, comparative fits to the entire field range as well as fits restricted to the low-field response lead to unphysical forms for $\sigma_{y x}^{\mathrm{SM}}(H)$.

[71] The full set of results obtained from this fitting procedure are summarized in Table I in Appendix E. In Table II, we compare the $\rho_{x x}^{\mathrm{NM}}(0)$ and $\rho_{x x}^{\mathrm{SM}}(0)$ values obtained from this analysis with those obtained from analysis of the MR in Ref. [18]. In most cases, the agreement is very good.

[72] The transformation of $\sigma_{y x}^{\mathrm{SM}}(H)$ to $\rho_{y x}^{\mathrm{SM}}(H)$ and the empirical relation are described in Appendix F.

[73] For completeness, the parameters obtained for the entire data set are listed in Table III in Appendix F.

[74] The extracted values are listed in Table IV in Appendix G.

[75] K. Kadowaki and S. B. Woods, Universal relationship of the resistivity and specific heat in heavy-fermion compounds, Solid State Commum. 58, 507 (1986).
[76] N. E. Hussey, Non-generality of the Kadowaki-Woods Ratio in correlated oxides, J. Phys. Soc. Jpn. 74, 1107 (2005).

[77] N. Doiron-Leyraud, C. Proust, D. LeBoeuf, J. Levallois, J.-B. Bonnemaison, R. Liang, D. A. Bonn, W. N. Hardy, and L. Taillefer, Quantum oscillations and the Fermi surface in an underdoped high- $T_{C}$ superconductor, Nature 447, 565 (2007).

[78] R. A. Borzi, S. A. Grigera, J. Farrell, R. S. Perry, S. J. S. Lister, S. L. Lee, D. A. Tennant, Y. Maeno, and A. P. Mackenzie, Formation of a nematic fluid at high fields in $\mathrm{Sr}_{3} \mathrm{Ru}_{2} \mathrm{O}_{7}$, Science 315, 214 (2007).

[79] C. Lester, S. Ramos, R. S. Perry, T. P. Croft, R. I. Bewley, T. Guidi, P. Manuel, D. D. Khalyavin, E. M. Forgan, and S. M. Hayden, Field-tunable spin-density-wave phases in $\mathrm{Sr}_{3} \mathrm{Ru}_{2} \mathrm{O}_{7}$, Nat. Mater. 14, 373-378 (2015).

[80] J.-F. Mercure, A. W. Rost, E. C. T. O'Farrell, S. K. Goh, R. S. Perry, M. L. Sutherland, S. A. Grigera, R. A. Borzi, P. Gegenwart, A. S. Gibbs, and A. P. Mackenzie, Quantum oscillations near the metamagnetic transition in $\mathrm{Sr}_{3} \mathrm{Ru}_{2} \mathrm{O}_{7}$, Phys. Rev. B 81, 235103 (2010).

[81] C. H. Mousatov, E. Berg, and S. A. Hartnoll, Theory of the strange metal $\mathrm{Sr}_{3} \mathrm{Ru}_{2} \mathrm{O}_{7}$, Proc. Natl. Acad. Sci. USA 117, 2852 (2020).

[82] J. Miao, X. H. Niu, D. F. Xu, Q. Yao, Q. Y. Chen, T. P. Ying, S. Y. Li, Y. F. Fang, J. C. Zhang, S. Ideta, K. Tanaka, B. P. Xie, D. L. Feng, and F. Chen, Electronic structure of FeS, Phys. Rev. B 95, 205127 (2017).

[83] T. Terashima, N. Kikugawa, D. Graf, H. T. Hirose, S. Uji, Y. Matsushita, H. Lin, X. Zhu, H.-H. Wen, T. Nomoto, K. Suzuki and H. Ikeda, Accurate determination of the Fermi surface of tetragonal $\mathrm{FeS}$ via quantum oscillation measurements and quasiparticle self-consistent $G W$ calculations, Phys. Rev. B 99, 134501 (2019).

[84] T. Chen, Y. Chen, A. Kreisel, X. Lu, A. Schneidewind, Y. Qiu, J. T. Park, T. G. Perring, J. R. Stewart, H. Cao, R. Zhang, Y. Li, Y. Rong, Y. Wei, B. M. Andersen, P. J. Hirschfeld, C. Broholm, and P. Dai, Anisotropic spin fluctuations in detwinned FeSe, Nat. Mater. 18, 709 (2019).

[85] N. E. Hussey, J. Buhot, and S. Licciardello, A tale of two metals: Contrasting criticalities in the pnictides and hole-doped cuprates, Rep. Prog. Phys. 81, 052501 (2018).

[86] X. Xu, A. Carrington, A. I. Coldea, A. Enayati-Rad, A. Narduzzo, S. Horii, and N. E. Hussey, Dimensionality-driven spin-flop transition in quasi-one-dimensional $\mathrm{PrBa}_{2} \mathrm{Cu}_{4} \mathrm{O}_{8}$, Phys. Rev. B 81, 224435 (2010).

[87] A. E. Böhmer, V. Taufour, W. E. Straszheim, T. Wolf, and P. C. Canfield, Variation of transition temperatures and residual resistivity ratio in vapor-grown FeSe, Phys. Rev. B 94, 024526 (2016).

[88] M. Ziman, Electrons and Phonons, Classics Series (Oxford University Press, Oxford, 2001). 